\title{
Integro-differential equations for option prices in exponential Lévy models ${ }^{1}$
}

\author{
Rama Cont and Ekaterina Voltchkova
}

Centre de Mathématiques Appliquées,

Ecole Polytechnique, F-91128 Palaiseau, France.

(e-mail: Rama.Cont@polytechnique.fr, voltchko@cmapx.polytechnique.fr )

\begin{abstract}
We explore the precise link between option prices in exponential Lévy models and the related partial integro-differential equations (PIDEs) in the case of European options and options with single or double barriers. We first discuss the conditions under which options prices are classical solutions of the PIDEs. We show that these conditions may fail in pure jump models and give examples of lack of smoothness of option prices with respect to the underlying. We give sufficient conditions on the Lévy triplet for the prices of barrier options to be continuous with respect to the underlying and show that, in a general setting, option prices in exp-Lévy models correspond to viscosity solutions of the pricing PIDE.
\end{abstract}

Key words: Lévy process, jump-diffusion models, option pricing, integrodifferential equations, viscosity solutions.

JEL Classification: G13

Mathematics Subject Classification (2000): 45K05, 49L25, 60G51, 60J75

The shortcomings of diffusion models in representing the risk related to large market movements have led to the development of various option pricing models with jumps, where large returns are represented as discontinuities of prices as a function of time. Models with jumps allow for more realistic representation of price dynamics and a greater flexibility in modelling and have been the focus of much recent work [9].

Exponential Lévy models, where the market price of an asset is represented as the exponential $S_{t}=\exp \left(r t+X_{t}\right)$ of a Lévy process $X_{t}$, offer analytically tractable examples of positive processes with jumps which are simple enough

\footnotetext{
${ }^{1}$ This work was presented at the Bachelier seminar (Paris, 2003), Workshop on Lévy processes and Partial integro-differential equations (Palaiseau, 2003), IFIP 2003 (Nice), the Workshop on Semimartingale theory and applications in finance (Banff) and the Workshop on Computational Finance (Zürich, 2003). We thank Yves Achdou, Mariko Arisawa, Daniel Gabay, Huyen Pham and Peter Tankov for helpful discussions.
} 
to allow a detailed study both in terms of statistical properties and as models for risk-neutral dynamics i.e. option pricing models. Option pricing with exponential Lévy models is discussed in $[9,14,16,19]$. The flexibility of choice of the Lévy process $X$ allows to calibrate the model to market prices of options and reproduce a wide variety of implied volatility skews/smiles. The Markov property of the price allows us to express option prices as solutions of partial integro-differential equations (PIDEs) which involve, in addition to a (possibly degenerate) second-order differential operator, a non-local integral term which requires specific treatment both at the theoretical and numerical level.

Such partial integro-differential equations (PIDEs) have been used by several authors to price options in models with jumps $[3,8,21,13]$ but the derivation of these equations is omitted in these works. We explore in this paper the precise link between option prices in exponential Lévy models and the related partial integro-differential equations (PIDEs) in the case of European and barrier options in exponential Lévy models. We first discuss the conditions under which options prices are classical solutions of the PIDEs and show that these conditions may fail in pure jump models, leading to a lack of smoothness with respect to the underlying. The notion of viscosity solution allows to cover this case: we give sufficient conditions on the Lévy triplet for the option price to be continuous and show that in this case it is a solution of the PIDE in the viscosity sense.

Section 1 recalls some basic facts about Lévy processes and exponential Lévy models. Section 2 derives the PIDE verified by option prices in a heuristic manner and discusses sufficient conditions for this derivation to hold. Section 3 gives two examples illustrating the lack of smoothness with respect to the underlying in pure jump models and gives sufficient conditions on the Lévy triplet for option prices to be continuous. In Section 4 we define the notion of viscosity solutions for PIDEs and give a characterization of option prices in terms of viscosity solution to a PIDE. Section 5 concludes by discussing relations with previous work, possible extensions and applications.

\section{Exponential Lévy models}

We consider here the class of models where the risk neutral dynamics of the underlying asset is given by $S_{t}=\exp \left(r t+X_{t}\right)$ where $X_{t}$ is a Lévy process.

\subsection{Lévy processes: definitions}

A Lévy process is a stochastic process $X_{t}$ with stationary independent increments. We set $X_{0}=0$. The characteristic function of $X_{t}$ has the following Lévy-Khinchin representation [26]:

$E\left[e^{i z X_{t}}\right]=\exp t \phi(z), \quad \phi(z)=-\frac{\sigma^{2} z^{2}}{2}+i \gamma z+\int_{-\infty}^{\infty}\left(e^{i z x}-1-i z x 1_{|x| \leq 1}\right) \nu(d x)$, 
where $\sigma \geq 0$ and $\gamma$ are real constants and $\nu$ is a positive Radon measure on $\mathbb{R} \backslash\{0\}$ verifying

$$
\int_{-1}^{+1} x^{2} \nu(d x)<\infty, \quad \int_{|x|>1} \nu(d x)<\infty
$$

The random process $X$ can be interpreted as the independent superposition of a Brownian motion with drift and an infinite superposition of independent (compensated) Poisson processes with various jump sizes $x, \nu(d x)$ being the intensity of jumps of size $x$. In general $\nu$ is not a finite measure: $\int \nu(d x)$ need not be finite.

A Lévy process is a (strong) Markov process $[6,26]$ : the associated semigroup is a convolution semigroup and its infinitesimal generator $L: f \rightarrow L f$ is an integro-differential operator given by:

$$
\begin{aligned}
L f(x) & =\lim _{t \rightarrow 0} \frac{E\left[f\left(x+X_{t}\right)\right]-f(x)}{t}= \\
& =\frac{\sigma^{2}}{2} \frac{\partial^{2} f}{\partial x^{2}}+\gamma \frac{\partial f}{\partial x}+\int \nu(d y)\left[f(x+y)-f(x)-y 1_{\{|y| \leq 1\}} \frac{\partial f}{\partial x}(x)\right]
\end{aligned}
$$

which is well defined for $f \in C^{2}(\mathbb{R})$ with compact support.

\subsection{Exponential Lévy models}

Let $\left(S_{t}\right)_{t \in[0, T]}$ be the price of a financial asset modelled as a stochastic process on a filtered probability space $\left(\Omega, \mathcal{F}, \mathcal{F}_{t}, \mathbb{P}\right) . \mathcal{F}_{t}$ is taken to be the price history up to $t$. Under the hypothesis of absence of arbitrage there exists a measure $\mathbb{Q}$ equivalent to $\mathbb{P}$ under which the discounted prices of all financial assets are $\mathbb{Q}$ martingales; in particular the discounted underlying $\left(e^{-r t} S_{t}\right)_{t \in[0, T]}$ is a martingale under $\mathbb{Q}$.

In exponential Lévy models, the (risk-neutral) dynamics of $S_{t}$ under $\mathbb{Q}$ is represented as the exponential of a Lévy process:

$$
S_{t}=S_{0} e^{r t+X_{t}}
$$

Here $X_{t}$ is a Lévy process (under $\mathbb{Q}$ ) with characteristic triplet $(\sigma, \gamma, \nu)$, and the interest rate $r$ is included for ease of notation. The absence of arbitrage then imposes that $\hat{S}_{t}=S_{t} e^{-r t}=\exp X_{t}$ is a martingale, which is equivalent to the following conditions on the triplet $(\sigma, \gamma, \nu)$ :

$$
\int_{|y|>1} \nu(d y) e^{y}<\infty, \quad \gamma=\gamma(\sigma, \nu)=-\frac{\sigma^{2}}{2}-\int\left(e^{y}-1-y 1_{|y| \leq 1}\right) \nu(d y) .
$$

We will assume (3) in the sequel. The infinitesimal generator $L$ then becomes:

$$
L f(x)=\frac{\sigma^{2}}{2}\left[\frac{\partial^{2} f}{\partial x^{2}}-\frac{\partial f}{\partial x}\right]+\int_{-\infty}^{\infty} \nu(d y)\left[f(x+y)-f(x)-\left(e^{y}-1\right) \frac{\partial f}{\partial x}(x)\right]
$$


The risk-neutral dynamics of $S_{t}$ is given by

$$
S_{t}=S_{0}+\int_{0}^{t} r S_{u-} d u+\int_{0}^{t} S_{u-} \sigma d W_{u}+\int_{0}^{t} \int_{-\infty}^{\infty}\left(e^{x}-1\right) S_{u-} \tilde{J}_{X}(d u d x)
$$

where $\tilde{J}_{X}$ is the compensated random measure describing the jumps of $X[17,24]$. $\left(S_{t}\right)$ is also a Markov process with state space $(0, \infty)$ and infinitesimal generator:

$$
L^{S} f(x)=r x \frac{\partial f}{\partial x}(x)+\frac{\sigma^{2} x^{2}}{2} \frac{\partial^{2} f}{\partial x^{2}}(x)+\int \nu(d y)\left[f\left(x e^{y}\right)-f(x)-x\left(e^{y}-1\right) \frac{\partial f}{\partial x}(x)\right] .
$$

While in principle one can have both a non-zero diffusion component $\sigma \neq 0$ and an infinite activity jump component, in practice the models encountered in the financial literature are of two types: either we combine a non-zero diffusion part $\sigma>0$ with a finite activity jump process (in this case one speaks of a jump-diffusion model) or one totally suppresses the diffusion part, in which case frequent small jumps are needed to generate realistic trajectories: these are infinite activity pure jump models [16, 19]. Different exponential Lévy models proposed in the financial modelling literature simply correspond to different choices for the Lévy measure $\nu$, see [9, Chap. 3] for a review.

\section{Integro-differential equations for option prices}

The value of a European option is defined as a discounted conditional expectation of its terminal payoff $H\left(S_{T}\right)$ under risk-neutral probability $\mathbb{Q}$ :

$$
C_{t}=E\left[e^{-r(T-t)} H\left(S_{T}\right) \mid \mathcal{F}_{t}\right] .
$$

From the Markov property, $C_{t}=C(t, S)$ where

$$
C(t, S)=E\left[e^{-r(T-t)} H\left(S_{T}\right) \mid S_{t}=S\right] .
$$

Introducing the change of variable $\tau=T-t, x=\ln \left(S / S_{0}\right)$, and defining: $h(x)=H\left(S_{0} e^{x}\right)$ and $f(\tau, x)=e^{r \tau} C\left(T-\tau, S_{0} e^{x}\right)$, then

$$
f(\tau, x)=E\left[h\left(x+r \tau+X_{\tau}\right)\right] .
$$

If $h$ is in the domain of the infinitesimal generator $L$ given by (4), then differentiating with respect to $\tau$ we obtain the following integro-differential equation:

$$
\frac{\partial f}{\partial \tau}=L f+r \frac{\partial f}{\partial x}, \quad \text { on }(0, T] \times \mathbb{R} ; \quad f(0, x)=h(x), \quad x \in \mathbb{R} .
$$

Similarly, if $f$ is smooth then using a change of variable we obtain a similar equation for $C(t, S)$ :

$$
\frac{\partial C}{\partial t}(t, S)+L^{S} C(t, S)-r C(t, S)=0 ; \quad C(T, S)=H(S) .
$$


This equation is similar to the Black-Scholes partial differential equation, except that the second-order differential operator is replaced by the integro-differential operators $L^{S}$.

However, the above reasoning is heuristic: the payoff function $h$ is usually not in the domain of $L$ and in fact it is usually not even differentiable. For example $h(x)=\left(K-S_{0} e^{x}\right)^{+}$for a put option and $h(x)=1_{x \geq x_{0}}$ for a binary option.

If $f$ is a smooth solution of (9), by applying the Ito formula to $f\left(t, X_{t}\right)$ between 0 and $T$ one can derive the probabilistic representation (8) for $f[5,22]$ :

Proposition 1 (Feynman-Kac representation for Lévy processes). Assume $\sigma>0$ or $\exists a>0$ such that $\int_{|x|>1} \exp (a|x|) \nu(d x)<\infty$. If $f \in C^{1,2}$ is a classical solution of (9) and its derivatives are bounded by a polynomial function of $x$, uniformly in $t \in[0, T]$, then $f$ has the probabilistic representation (8).

The case $\sigma>0$ is shown in [5, Chap. 4]; the pure jump case is treated in [22]. This type of result is sometimes called a verification theorem: $f$ is assumed to be smooth and its derivatives assumed to verify some integrability conditions. The conditions on $f$ and $\nu$ ensure that $f\left(t, X_{t}\right)$ can be represented as a martingale plus a finite variation process. However, it is readily seen that such conditions are never verified in option pricing applications. ${ }^{2}$ For instance, even for a European put option, the second derivative (Gamma of the option) is certainly not uniformly bounded in $t$ !

These assumptions can be weakened in various ways [5, 25]. In the next section we will give some sufficient conditions for the option to be a classical solutions. Under these conditions the value of European options $f(\tau, x), C(t, S)$ defined above are classical solutions of the partial integro-differential equations (9), (10). However, as we will see in section 3, these conditions are not always verified, especially in pure jump models. This will lead us to consider the notion of viscosity solution; we show in section 4 that under more general conditions, values of European or barrier options can be expressed as viscosity solutions of appropriate PIDEs.

\subsection{Classical solutions}

Consider a European option with maturity $T$ and payoff $H\left(S_{T}\right)$. Assume that the payoff function $H$ is Lipschitz:

$$
|H(x)-H(y)| \leq c|x-y|
$$

for some $c>0$. This condition is of course verified by call and put options with $c=1$. The value $C_{t}$ of such an option is given by $C_{t}=C\left(t, S_{t}\right)$ where

$$
C(t, S)=e^{-r(T-t)} E\left[H\left(S_{T}\right) \mid S_{t}=S\right]=e^{-r(T-t)} E\left[H\left(S e^{r(T-t)+X_{T-t}}\right)\right] .
$$

We will furthermore assume, throughout this section, that

$$
\int_{|y|>1} e^{2 y} \nu(d y)<\infty
$$

\footnotetext{
${ }^{2}$ In particular, the hypotheses in [22] do not apply to the example of a call or put option.
} 
This condition is equivalent to the existence of a second moment for the price process $S_{t}$. Then $\hat{S}_{t}=\exp X_{t}$ is a square integrable martingale:

$$
\frac{d \hat{S}_{t}}{\hat{S}_{t-}}=\sigma d W_{t}+\int_{-\infty}^{\infty}\left(e^{x}-1\right) \tilde{J}_{X}(d t d x), \quad \sup _{t \in[0, T]} E\left[\hat{S}_{t}^{2}\right]<\infty .
$$

Proposition 2. Consider the exponential Lévy model $S_{t}=S_{0} \exp \left(r t+X_{t}\right)$ where the Lévy process $X$ verifies (12). If

$$
\sigma>0 \quad \text { or } \quad \exists \beta \in(0,2), \quad \liminf _{\epsilon \downarrow 0} \epsilon^{-\beta} \int_{-\epsilon}^{\epsilon}|x|^{2} \nu(d x)>0
$$

then the value of a terminal payoff $H\left(S_{T}\right)$ is given by $C(t, S)$ where:

$$
\begin{aligned}
C:[0, T] \times[0, \infty) & \rightarrow \mathbb{R} \\
(t, S) & \mapsto C(t, S)=e^{-r(T-t)} E\left[H\left(S_{T}\right) \mid S_{t}=S\right]
\end{aligned}
$$

is continuous on $[0, T] \times[0, \infty), C^{1,2}$ on $(0, T) \times(0, \infty)$, and verifies the partial integro-differential equation:

$$
\begin{aligned}
\frac{\partial C}{\partial t}(t, S)+r S & \frac{\partial C}{\partial S}(t, S)+\frac{\sigma^{2} S^{2}}{2} \frac{\partial^{2} C}{\partial S^{2}}(t, S)-r C(t, S)+ \\
& +\int \nu(d x)\left[C\left(t, S e^{x}\right)-C(t, S)-S\left(e^{x}-1\right) \frac{\partial C}{\partial S}(t, S)\right]=0
\end{aligned}
$$

on $[0, T) \times(0, \infty)$ with the terminal condition:

$$
\forall S>0, \quad C(T, S)=H(S) .
$$

Proof. The proof involves, as in the Black-Scholes case, applying the Itô formula to the martingale $\hat{C}\left(t, S_{t}\right)=e^{r(T-t)} C\left(t, S_{t}\right)$, identifying the drift component and setting it to zero.

Condition (13) implies that $X_{t}$ has a smooth $C^{2}$ density with derivatives vanishing at infinity [26, Prop. 28.3]; $C(t, S)$ is then a smooth function of $S$. Smoothness in time can be shown by Fourier methods, see e.g. [11, Appendix 4]. By construction, $\hat{C}_{t}=E\left[H \mid \mathcal{F}_{t}\right]$ is a martingale. Applying the Itô formula to $\hat{C}_{t}=e^{r(T-t)} C\left(t, S_{t}\right)$ and using equation (5) we obtain:

$$
\begin{aligned}
d \hat{C}_{t}= & e^{r(T-t)}\left[-r C_{t}+\frac{\partial C}{\partial t}\left(t, S_{t-}\right)+\frac{\sigma^{2} S_{t}^{2}}{2} \frac{\partial^{2} C}{\partial S^{2}}\left(t, S_{t-}\right)\right] d t+e^{r(T-t)} \frac{\partial C}{\partial S}\left(t, S_{t-}\right) d S_{t}+ \\
& +e^{r(T-t)}\left[C\left(t, S_{t-} e^{\Delta X_{t}}\right)-C\left(t, S_{t-}\right)-S_{t-}\left(e^{\Delta X_{t}}-1\right) \frac{\partial C}{\partial S}\left(t, S_{t-}\right)\right]= \\
& =a(t) d t+d M_{t} \quad(16)
\end{aligned}
$$

where

$$
\begin{aligned}
a(t) & =e^{r(T-t)}\left[-r C\left(t, S_{t-}\right)+\frac{\partial C}{\partial t}\left(t, S_{t-}\right)+\frac{\sigma^{2} S_{t-}^{2}}{2} \frac{\partial^{2} C}{\partial S^{2}}\left(t, S_{t-}\right)+r S_{t-} \frac{\partial C}{\partial S}\left(t, S_{t-}\right)\right] \\
& +\int_{-\infty}^{\infty} \nu(d x) e^{r(T-t)}\left[C\left(t, S_{t-} e^{x}\right)-C\left(t, S_{t-}\right)-S_{t-}\left(e^{x}-1\right) \frac{\partial C}{\partial S}\left(t, S_{t-}\right)\right]
\end{aligned}
$$


and

$$
d M_{t}=e^{r(T-t)} \frac{\partial C}{\partial S}\left(t, S_{t-}\right) \sigma S_{t-} d W_{t}+\int_{\mathbb{R}} e^{r(T-t)}\left[C\left(t, S_{t-} e^{x}\right)-C\left(t, S_{t-}\right)\right] \tilde{J}_{X}(d t d x) .
$$

Let us show that $M_{t}$ is a martingale. Since the payoff function $H$ is Lipschitz, $C$ is also Lipschitz with respect to the second variable:

$$
\begin{aligned}
\left|C\left(t, S_{1}\right)-C\left(t, S_{2}\right)\right| & =e^{-r(T-t)}\left|E\left[H\left(S_{1} e^{r(T-t)+X_{T-t}}\right)\right]-E\left[H\left(S_{2} e^{r(T-t)+X_{T-t}}\right)\right]\right| \\
& \leq c\left|S_{1}-S_{2}\right| E\left[e^{X_{T-t}}\right]=c\left|S_{1}-S_{2}\right|
\end{aligned}
$$

since $e^{X_{t}}$ is a martingale. Therefore the predictable random function $\psi(t, x)=$ $C\left(t, S_{t-} e^{x}\right)-C\left(t, S_{t-}\right)$ verifies

$$
\begin{aligned}
E\left[\int_{0}^{T} d t \int_{\mathbb{R}} \nu(d x)|\psi(t, x)|^{2}\right] & =E\left[\int_{0}^{T} d t \int_{\mathbb{R}} \nu(d x)\left|C\left(t, S_{t-} e^{x}\right)-C\left(t, S_{t-}\right)\right|^{2}\right] \\
& \leq E\left[\int_{0}^{T} d t \int_{\mathbb{R}} c^{2}\left(e^{2 x}+1\right) S_{t-}^{2} \nu(d x)\right] \\
\operatorname{using}(12) & \leq c^{2} \int_{\mathbb{R}}\left(e^{2 x}+1\right) \nu(d x) E\left[\int_{0}^{T} S_{t-}^{2} d t\right]<\infty,
\end{aligned}
$$

so the compensated Poisson integral

$$
\int_{0}^{t} \int_{-\infty}^{\infty} e^{r(T-t)}\left[C\left(t, S_{t-} e^{x}\right)-C\left(t, S_{t-}\right)\right] \tilde{J}_{X}(d t d x)
$$

is a square integrable martingale. Also, since $C$ is Lipschitz, $\partial C / \partial S \in L^{\infty}$ and

$$
\left\|\frac{\partial C}{\partial S}(t, .)\right\|_{L^{\infty}} \leq c, \quad \text { so } \quad E\left[\int_{0}^{T} S_{t-\mid}^{2}\left|\frac{\partial C}{\partial S}\left(t, S_{t-}\right)\right|^{2} d t\right] \leq c^{2} E\left[\int_{0}^{T} S_{t-}^{2} d t\right]<\infty .
$$

Using the isometry relation for Wiener integrals, we obtain that $\int_{0}^{t} \sigma S_{t} \frac{\partial C}{\partial S}\left(t, S_{t-}\right) d W_{t}$ is also a square integrable martingale. Therefore $M_{t}$ is a square integrable martingale. $\hat{C}_{t}-M_{t}$ is thus a (square integrable) martingale; but $\hat{C}_{t}-M_{t}=\int_{0}^{t} a(s) d s$ is also a continuous process with finite variation so, by [17, Theorem 4.13-4.50], we must have $a(t)=0 \mathbb{Q}$-almost surely which yields the PIDE (14).

The condition (13) holds for all jump diffusion models with non-zero diffusion component as well as for Lévy densities behaving near zero as $\nu(x) \sim c / x^{1+\beta}$ with $\beta>0$ such as the tempered stable model, but not for the Variance Gamma model [20]. In the case of the Variance Gamma model, the PIDE reduces to a first order equation for which only $C^{1}$ smoothness is required but, as we shall observed in Section 3, even this condition may fail. 


\section{$2.2 \quad$ Barrier options}

Barrier options can be similarly represented in terms of solutions to PIDEs. Consider for instance an up-and-out call option with maturity $T$, strike $K$, and (upper) barrier $U>S_{0}$. The terminal payoff is given by

$$
H_{T}=\left(S_{T}-K\right)^{+} 1_{T<\theta}
$$

where $\theta=\inf \left\{t \geq 0 \mid S_{t} \geq U\right\}$, the first moment when the barrier is crossed.

The value of the barrier option at time $t$ is defined as the discounted expectation of it's terminal payoff: $C_{t}=e^{-r(T-t)} E\left[H_{T} \mid \mathcal{F}_{t}\right]$. By construction, $e^{r(T-t)} C_{t}$ is a martingale.

Due to the Markov property of Lévy processes, it is possible to express the price $C_{t}$ as a deterministic function of time $t$ and current stock value $S_{t}$ before the barrier is crossed. Namely, for any $(t, S) \in[0, T] \times(0, \infty)$ we can define

$$
C_{b}(t, S)=e^{-r(T-t)} E\left[H\left(S e^{Y_{T-t}}\right) 1_{T<\theta_{t}}\right],
$$

where $H(S)=(S-K)^{+},\left\{Y_{s-t}, s \geq t\right\}$ is a Lévy process, and $\theta_{t}=\inf \{s \geq$ $\left.t \mid S e^{Y_{s-t}} \geq U\right\}$, the first exit time after $t$. Then

$$
C_{t}=C_{b}\left(t, S_{t}\right) 1_{t \leq \theta}
$$

for all $t \leq T$. Note that outside of the set $\{t \leq \theta\}$ the objects $C_{t}$ and $C_{b}\left(t, S_{t}\right)$ are different: if the barrier has already been crossed, $C_{t}$ will always be zero, but $C_{b}\left(t, S_{t}\right)$ may become positive if the stock returns to the region below the barrier. By going to the log variables we define

$$
f_{b}(\tau, x)=e^{r \tau} C_{b}\left(T-\tau, S_{0} e^{x}\right) .
$$

Again, if $f_{b}$ is smooth the Itô formula can be used to show that $f_{b}$ is a solution of the following initial-boundary-value problem:

$$
\begin{array}{ll}
\frac{\partial f}{\partial \tau}=L f+r \frac{\partial f}{\partial x}, & \text { on }(0, T] \times\left(-\infty, \log \left(U / S_{0}\right)\right), \\
f(0, x)=h(x), & x<\log \left(U / S_{0}\right), \\
f(\tau, x)=0, & x \geq \log \left(U / S_{0}\right) .
\end{array}
$$

The main difference between this equation and the analogous PDEs for diffusion models is in the "boundary condition": (22) not only specifies the behavior of the solution at the barrier $S=U$ but also beyond the barrier $(S>U)$. This is necessary because of the non-local nature of the operator $L$ : to compute the integral term we need the function $f(\tau,$.$) on (-\infty, \infty)$ and $(22)$ extends the function beyond the barrier by zero. In the case of a rebate, the function would be replaced by the value of the rebate in the knock-out region $S \geq U$. Similar results and corresponding Feynman-Kac formulae hold — in the case $\sigma>0$ when the boundary condition is not zero but given by a function $g(\tau, x)$ where $g \in W_{p}^{1,2}([0, T] \times \mathbb{R})$ with $p>3$, see $[25]$. More generally, if $f_{b}(.,$.$) defined by (20)$ can be shown to be $C^{1,2}$ (or simply $C^{1,1}$ in the case of finite variation models) 
then following the proof of Proposition 2, one can show that $f_{b}$ is a solution of the PIDE (21). However, as we shall see below (Example 2) in the case of pure jump models where $\sigma=0$ such smoothness with respect to the underlying does not hold in general.

\section{Smoothness with respect to the underlying}

In the case where the log-price $X_{t}$ has a non-degenerate diffusion component, it is known $[5,15]$ that the fundamental solution of the pricing PIDE, which corresponds to the density of $X_{t}$, is in fact a smooth $C^{\infty}$ function. As a consequence, the option price $u(t, x)$ depends smoothly on the underlying and results such as Proposition 1 allow to use the solution of the PIDE to compute the option price. In pure jump models, this property may fail. In this section we present examples where smoothness fails; we then give sufficient conditions under which the option price is continuous as a function of the underlying. This minimal regularity will be required later to show that the option price is a generalized (viscosity) solution of the PIDE.

\subsection{Lack of smoothness in pure jump models}

In the case of processes with a degenerate diffusion component, such as pure jump models, the smoothness of the conditional expectation as a function of the initial (spot) value of the underlying $S$ does not always hold, as the following example shows.

Example 1 (Variance Gamma process). The Variance Gamma process, introduced by Madan \& Milne [20], is a pure jump finite variation process with infinite activity, popular in financial modelling. Its Lévy measure has a density given by:

$$
\nu(x)=\frac{1}{\kappa|x|} e^{A x-B|x|} \quad \text { with } \quad A=\frac{\theta}{\sigma^{2}} \text { and } B=\frac{\sqrt{\theta^{2}+2 \sigma^{2} / \kappa}}{\sigma^{2}} .
$$

The characteristic function of $X_{t}$ is given by:

$$
\Phi_{t}(u)=\left(1+\frac{u^{2} \sigma^{2} \kappa}{2}-i \theta \kappa u\right)^{-\frac{t}{\kappa}}
$$

Since $\Phi_{t}$ is the Fourier transform of the distribution of $X_{t}$, the smoothness of the distribution can be read from the rate of decay of $\Phi_{t}(u)$ when $|u| \rightarrow \infty$. In this case, $\Phi_{t}($.$) decays as |u|^{-2 t / \kappa}$ when $|u| \rightarrow \infty$ : the decay exponent increases with $t$. The fundamental solution $\rho(t, x)$ of the PIDE therefore has a degree of regularity which increases gradually with $t$ : for $t \in(p \kappa / 2,(p+1) \kappa / 2)$, the fundamental solution $\rho(t,$.$) is in C^{p-1}(\mathbb{R})$ but not $C^{p}(\mathbb{R})$. For $t<\kappa / 2, \rho(t,$.$) is$ not even locally bounded. Consider now the value of a European binary option defined by the payoff $h(x)=1_{x>x_{0}}$ : its value is shown in figure 1 . Being the primitive of $\rho(t,$.$) , its value is continuous but not differentiable in x$ for $t<\kappa / 2$ : the option price has a vertical tangent at the money. 


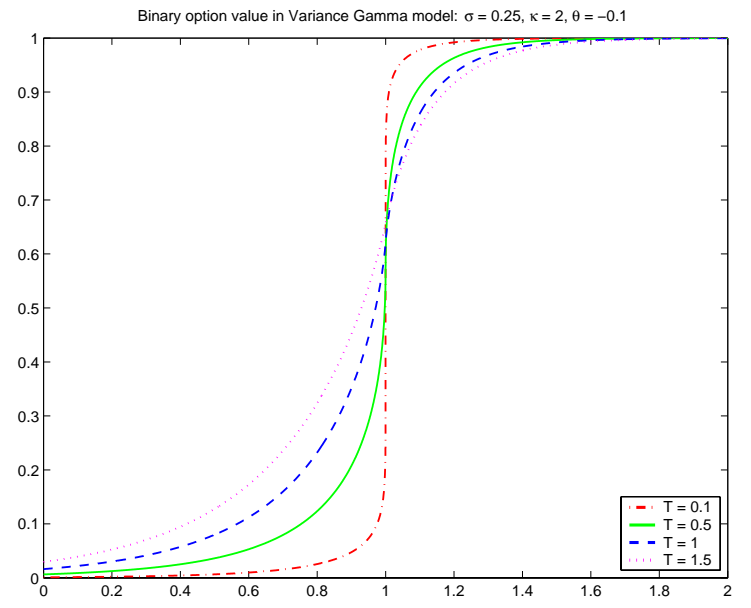

Figure 1: Value of a binary option in the Variance Gamma model, as a function of the underlying.

The case of barrier options is even less regular. As the following example illustrates, if no restriction is imposed on the Lévy process, the value of a barrier option - which is formally the solution of the Dirichlet problem with zero boundary conditions - can even turn out to be discontinuous at all times:

Example 2. Consider $X_{t}=N_{t}^{1}-N_{t}^{2}$ where $N_{t}^{i}$ are independent Poisson processes with jump intensities $\lambda_{1}$ and $\lambda_{2}$. Let, for simplicity, $r=0$. If $\lambda_{2}=\lambda_{1} e$ then the corresponding price process $S_{t}=S_{0} e^{X_{t}}$ is a martingale.

Consider now a knock-out option which pays 1 at time $T$ if $S_{t}$ has not crossed the barrier $U>S_{0}$ before $T$, and 0 otherwise:

$$
H_{T}=1_{T<\theta\left(S_{0}\right)},
$$

where $\theta(S)=\inf \left\{t \geq 0 \mid S e^{X_{t}} \geq U\right\}$ is the first exit time if the process starts from $S$. Let us show that the initial option value

$$
C(0, S)=\mathbb{E}\left[H_{T} \mid S_{0}=S\right]=\mathbb{E}\left[1_{T<\theta(S)}\right]
$$

is not continuous at $S^{*}=U / e$. Let $0<\varepsilon<U-S^{*}$. By definition, $\theta\left(S^{*}+\varepsilon\right) \leq$ $\theta\left(S^{*}-\varepsilon\right)$. Therefore,

$$
\begin{aligned}
C\left(0, S^{*}-\varepsilon\right)-C\left(0, S^{*}+\varepsilon\right) & =\mathbb{E}\left[1_{\left\{\theta\left(S^{*}+\varepsilon\right) \leq T<\theta\left(S^{*}-\varepsilon\right)\right\}}\right] \\
& =\mathbb{Q}\left(\theta\left(S^{*}+\varepsilon\right) \leq T<\theta\left(S^{*}-\varepsilon\right)\right) .
\end{aligned}
$$

Consider the event where there is one positive and no negative jumps $\left\{N_{T}^{1}=\right.$ $\left.1, N_{T}^{2}=0\right\}$, which has non-zero probability. In this case, if $S_{t}$ starts from $S^{*}-\varepsilon$ it stays below $U$, while starting from $S^{*}+\varepsilon$ it crosses the barrier. This means that $\theta\left(S^{*}+\varepsilon\right) \leq T<\theta\left(S^{*}-\varepsilon\right)$. So,

$$
C\left(0, S^{*}-\varepsilon\right)-C\left(0, S^{*}+\varepsilon\right) \geq \mathbb{Q}\left(N_{T}^{1}=1 \& N_{T}^{2}=0\right)=e^{-\lambda_{1} T(e+1)} \lambda_{1} T>0 .
$$


Thus $S \mapsto C(0, S)$ is discontinuous at $S=S^{*}$.

This example is a finite activity process without diffusion component. As noted above, this case is not the interesting one in financial modelling. In the next section, we show that in fact, in most cases of interest, the option price is a continuous function of the underlying.

\subsection{Continuity with respect to the underlying}

We start with showing that the value of a European option is a continuous function of its arguments, under the Lipschitz condition on the payoff and without any additional restriction on the Lévy density. Next, we give sufficient conditions on the Lévy triplet of $X$ which guarantee the continuity of values of barrier options.

\section{Proposition 3. (Continuity of European options)}

If $H$ satisfies the Lipschitz condition (11) then the forward value of a European option defined by (8): $f(\tau, x)=\mathbb{E}\left[H\left(S_{0} e^{x+r \tau+X_{\tau}}\right)\right]$ is continuous on $[0, T] \times \mathbb{R}$.

Proof. The continuity in $x$ is straightforward:

$$
\begin{aligned}
|f(\tau, x+\Delta x)-f(\tau, x)| & =\left|\mathbb{E}\left[H\left(S_{0} e^{x+\Delta x+r \tau+X_{\tau}}\right)\right]-\mathbb{E}\left[H\left(S_{0} e^{x+r \tau+X_{\tau}}\right)\right]\right| \\
& \leq c S_{0} e^{x+r \tau}\left|e^{\Delta x}-1\right| \mathbb{E}\left[e^{X_{\tau}}\right] \rightarrow 0 \quad \text { as }|\Delta x| \rightarrow 0,
\end{aligned}
$$

since $\mathbb{E} e^{X_{\tau}}=1$ by the martingale condition. To show continuity in $t$, let $t \geq$ $s \geq 0$ (the case $s \geq t$ is symmetrical). Then, $X_{t} \stackrel{d}{=} X_{s}+X_{t-s}, X_{t-s} \Perp X_{s}$ and we obtain

$$
\begin{aligned}
|f(t, x)-f(s, x)| & \leq \mathbb{E}\left[\left|H\left(S_{0} e^{x+r t+X_{t}}\right)-H\left(S_{0} e^{x+r s+X_{s}}\right)\right|\right] \\
& \leq c S_{0} e^{x+r s} \mathbb{E}\left|e^{r(t-s)+X_{t-s}}-1\right| .
\end{aligned}
$$

So, we need to show that $\mathbb{E}\left|e^{r \tau+X_{\tau}}-1\right| \rightarrow 0$ as $\tau \rightarrow 0$. First, we remark that the martingale condition implies:

$$
\mathbb{E}\left|e^{r \tau+X_{\tau}}-1\right|=e^{r \tau}-1+2 \mathbb{E}\left[\left(1-e^{r \tau+X_{\tau}}\right)^{+}\right] .
$$

Let $C_{0}(\mathbb{R})$ be the set of continuous functions vanishing at infinity. By the Feller property for any $g \in C_{0}(\mathbb{R})$, we have

$$
P_{\tau} g(0) \equiv \mathbb{E} g\left(r \tau+X_{\tau}\right) \stackrel{\tau \downarrow 0}{\longrightarrow} g(0),
$$

where $P_{\tau}$ is the semigroup of the process $\left\{r \tau+X_{\tau}\right\}$. Since $g(x)=\left(1-e^{x}\right)^{+}$is not in $C_{0}(\mathbb{R})$, we approximate it with a function $\tilde{g}(x)$, such that

$$
\begin{aligned}
& \tilde{g}(x)=g(x), \text { if } x \geq-1, \\
& \tilde{g}(x)=0, \text { if } x \leq-2, \\
& 0 \leq \tilde{g}(x) \leq g(x),
\end{aligned}
$$


and $\tilde{g}(x)$ is continuously interpolated between -2 and -1 . By construction, $\tilde{g} \in C_{0}(\mathbb{R})$. We obtain

$$
\begin{aligned}
\mathbb{E}\left[\left(1-e^{r \tau+X_{\tau}}\right)^{+}\right] & =\left|P_{\tau} g(0)\right| \leq\left|P_{\tau} g(0)-P_{\tau} \tilde{g}(0)\right|+\left|P_{\tau} \tilde{g}(0)\right| \\
& =\mathbb{E}\left[g\left(r \tau+X_{\tau}\right)-\tilde{g}\left(r \tau+X_{\tau}\right)\right]+\left|P_{\tau} \tilde{g}(0)\right| \\
& =\mathbb{E}\left[\left(g\left(r \tau+X_{\tau}\right)-\tilde{g}\left(r \tau+X_{\tau}\right)\right) 1_{\left\{r \tau+X_{\tau}<-1\right\}}\right]+\left|P_{\tau} \tilde{g}(0)\right| \\
& \leq \mathbb{Q}\left[r \tau+X_{\tau}<-1\right]+\left|P_{\tau} \tilde{g}(0)\right| \leq \mathbb{Q}\left[X_{\tau} \leq-1\right]+\left|P_{\tau} \tilde{g}(0)\right|,
\end{aligned}
$$

since $g(x) \leq 1$. By (26), we have $\left|P_{\tau} \tilde{g}(0)\right| \rightarrow 0$ as $\tau \rightarrow 0$. So, the last point to show is that $\mathbb{Q}\left[X_{\tau} \leq-1\right] \rightarrow 0$ as $\tau \rightarrow 0$.

Defining $M_{\tau}^{-}=\sup _{0 \leq s<\tau}\left(-X_{s}\right)$, we have $\mathbb{Q}\left[X_{\tau} \leq-1\right] \leq \mathbb{Q}\left[M_{\tau}^{-} \geq 1\right]$. Let us take a sequence $\tau_{n} \downarrow 0$ and define $\Omega_{n}=\left\{\omega \in \Omega \mid M_{\tau_{n}}^{-}(\omega) \geq 1\right\}$. The sequence $\left\{\Omega_{n}\right\}$ is decreasing and

$$
\bigcap_{n>0} \Omega_{n}=\left\{\omega \in \Omega \mid \forall n, M_{\tau_{n}}^{-}(\omega) \geq 1\right\} \subseteq\left\{\omega \in \Omega \mid M_{0}^{-}(\omega) \geq 1\right\},
$$

by the right-continuity of $X_{\tau}$. Since $M_{0}^{-}=X_{0}=0$, we obtain

$$
\mathbb{Q}\left[M_{\tau_{n}}^{-} \geq 1\right]=\mathbb{Q}\left(\Omega_{n}\right) \stackrel{\tau_{n} \downarrow 0}{\longrightarrow} \mathbb{Q}\left(\bigcap \Omega_{n}\right)=0, \quad \text { so } \mathbb{Q}\left[M_{\tau}^{-} \geq 1\right] \rightarrow 0
$$

since $\left\{\tau_{n}\right\}$ is arbitrary. Therefore, $\mathbb{Q}\left[X_{\tau} \leq-1\right] \rightarrow 0$.

To study the continuity of barrier options we extensively use the properties of the first passage time process. Following the notation of [26], we define

$$
\begin{aligned}
& R_{x}=\inf \left\{s \geq 0 \mid Y_{s}>x\right\}, \\
& R_{x}^{\prime \prime}=\inf \left\{s \geq 0 \mid Y_{s} \vee Y_{s-} \geq x\right\} .
\end{aligned}
$$

Note that $\left\{R_{x}, x \geq 0\right\}$ is a process with non-decreasing paths, so we can define $R_{x-}(\omega)=\lim _{\varepsilon \downarrow 0} R_{x-\varepsilon}(\omega)$. Since $Y_{t}$ is right-continuous, the process $R_{x}$ is also right-continuous in $x$. We use the following terminology [26]:

Definition 1. A Lévy process with generating triplet $(\sigma, \gamma, \nu)$ is said to be of

type A (compound Poisson) if $\sigma=0$ and $\nu(\mathbb{R})<\infty$

type $\mathbf{B}$ (finite variation, infinite intensity) if $\sigma=0, \nu(\mathbb{R})=\infty$ and $\int_{|x| \leq 1}|x| \nu(d x)<\infty$

type C (infinite variation) if $\sigma>0$ or $\int_{|x| \leq 1}|x| \nu(d x)=\infty$.

Now we give some properties of the process $\left\{R_{x}\right\}$ which are essential to prove the continuity of option values.

Lemma 1. If $\left\{Y_{t}\right\}$ is of type $B$ or $C$ then:

$$
\forall x>0, \quad \mathbb{Q}\left[R_{x-}=R_{x}\right]=1 .
$$


Proof. For a fixed $x>0$, we introduce two subsets of $\Omega$ :

$$
\Omega_{1}=\left\{\omega \mid R_{x-}(\omega)<R_{x}(\omega)\right\}, \quad \Omega_{2}=\left\{\omega \mid R_{x}(\omega)=R_{x}^{\prime \prime}(\omega)\right\} .
$$

By the Lemma 49.6 of [26], $\mathbb{Q}\left(\Omega_{2}\right)=1$. So, it suffices to show that $\Omega_{1} \cap \Omega_{2}=\emptyset$, since, by definition, $R_{x-} \leq R_{x}$.

If there exists $\omega \in \Omega_{1} \cap \Omega_{2}$ then for the sample path $R_{x}=R_{x}(\omega)$ we have $R_{x-}<R_{x}^{\prime \prime}$. Therefore,

$$
\begin{aligned}
& \exists u \geq 0, R_{x-}=u, \\
& \exists t>u, R_{x}^{\prime \prime}=t .
\end{aligned}
$$

The definition of $R_{x-}$ together with (28) implies that

$$
\forall \varepsilon>0, \forall \delta>0, \exists s<u+\delta: Y_{s}>x-\varepsilon .
$$

Take $\varepsilon_{n}=\delta_{n}=1 / n$. Then, there exists a sequence $\left\{s_{n}\right\}$, such that $\forall n$,

$$
s_{n}<u+1 / n, \quad Y_{s_{n}}>x-1 / n .
$$

Since $\left\{s_{n}\right\}$ is bounded, one can extract a convergent subsequence $s_{n} \uparrow s_{0}$ or $s_{n} \downarrow s_{0}$, with $s_{0} \leq u<t$. In the first case we obtain $Y_{s_{0}-} \geq x$ and in the second, $Y_{s_{0}} \geq x$. So, in all cases, $Y_{s_{0}-} \vee Y_{s_{0}} \geq x$. But (29) implies

$$
\forall s<t, \quad Y_{s-} \vee Y_{s}<x .
$$

This contradiction proves that $\Omega_{1} \cap \Omega_{2}=\emptyset$, hence $\mathbb{Q}\left(\Omega_{1}\right)=0$.

An important property of $\left\{R_{x}\right\}$ is whether $R_{0}=0$ a.s.. Table 1 , which is a consequence of Theorem 47.5 of [26], relates this property to properties of the Lévy triplet for different types of Lévy processes.

Define now the supremum process of $Y$ :

$$
M_{t}=\sup _{0 \leq s \leq t} Y_{s}
$$

$M_{t}$ is non-decreasing and càdlàg, since $Y_{t}$ is càdlàg.

Lemma 2. If $\left\{Y_{t}\right\}$ is of type $B$ with $R_{0}=0$ a.s., or of type $C$, then:

$$
\forall x>0, \quad \forall t \geq 0, \quad \mathbb{Q}\left[R_{x}=t\right]=0 .
$$

Proof. By the definition of $R_{x}$,

$$
\begin{aligned}
\mathbb{Q}\left[R_{x}=t\right] & =\mathbb{Q}\left[\forall s<t, Y_{s} \leq x ; \exists s_{n} \downarrow t, Y_{s_{n}}>x\right] \\
& \leq \mathbb{Q}\left[M_{t-} \leq x ; M_{t} \geq x\right] .
\end{aligned}
$$

From Lemma 49.3 of $[26], \forall t>0, \forall x \geq 0, \mathbb{Q}\left[M_{t}=x\right]=0$ therefore

$$
\mathbb{Q}\left[R_{x}=t\right] \leq \mathbb{Q}\left[M_{t-} \leq x<M_{t}\right] \leq \mathbb{Q}\left[M_{t-} \neq M_{t}\right] \leq \mathbb{Q}\left[Y_{t-} \neq Y_{t}\right]=0,
$$

since a Lévy process has, almost surely, no fixed times of discontinuity. For the same reason, $\forall x>0, \mathbb{Q}\left[R_{x}=0\right]=0$, which completes the proof. 
Lemma 3. If $\left\{Y_{t}\right\}$ is of type $B$ or $C$, then $\forall t \geq 0, \forall x>0$,

$$
\begin{aligned}
& \mathbb{Q}\left[R_{x} \leq t<R_{x+\varepsilon}\right] \rightarrow 0, \\
& \mathbb{Q}\left[R_{x-\varepsilon} \leq t<R_{x}\right] \rightarrow 0,
\end{aligned}
$$

as $\varepsilon \downarrow 0$. If, in addition, $R_{0}=0$ a.s. then (30) is also satisfied for $x=0, t>0$.

Proof. For all fixed $t \geq 0, x \geq 0$, the sequence $\Omega_{\varepsilon}=\left\{\omega \in \Omega \mid R_{x}(\omega) \leq t<\right.$ $\left.R_{x+\varepsilon}(\omega)\right\}$ is decreasing and $\bigcap \Omega_{\varepsilon}=\left\{\omega \in \Omega \mid R_{x}(\omega)=t\right\}$, by the right-continuity of $R_{x}$. Therefore

$$
\mathbb{Q}\left[R_{x} \leq t<R_{x+\varepsilon}\right] \rightarrow \mathbb{Q}\left[R_{x}=t\right] .
$$

If $x>0$, this probability is zero by the Lemma 2. If $R_{0}=0$ a.s. then, $\forall t>0$, $\mathbb{Q}\left[R_{0}=t\right]=0$.

Similarly, for all $t \geq 0, x>0$,

$$
\mathbb{Q}\left[R_{x-\varepsilon} \leq t<R_{x}\right] \rightarrow \mathbb{Q}\left[R_{x-} \leq t<R_{x}\right] \leq \mathbb{Q}\left[R_{x-} \neq R_{x}\right]=0,
$$

by the Lemma 1.

$Y_{t}=r t+X_{t}$ is of the same type as $\left\{X_{t}\right\}$ but the property of $R_{0}$, which in the finite-variation case depends on the drift, is not necessary the same for the two processes. Therefore, it is worth noting that $\left\{R_{x}\right\}$ will be always defined with respect to $\left\{Y_{t}\right\}$. Now we are in a position to consider the continuity of value functions for barrier options. We start with the case of a single upper barrier $U>S_{0}$.

\section{Proposition 4. (Continuity of up-and-out options)}

Let $Y_{t}$ be of type $B$ or $C$ and $R_{0}=0$ a.s. Assume that $H:(0, U) \rightarrow[0, \infty)$ is Lipschitz:

$$
\forall S_{1}, S_{2} \in(0, U), \quad\left|H\left(S_{1}\right)-H\left(S_{2}\right)\right| \leq c\left|S_{1}-S_{2}\right|,
$$

with $c>0$ and denote $u=\log \left(U / S_{0}\right)$. Then, the function

$$
f_{u}(\tau, x)= \begin{cases}\mathbb{E}\left[H\left(S_{0} e^{x+Y_{\tau}}\right) 1_{\left\{\tau<R_{u-x}\right\}}\right], & x<u, \\ 0, & x \geq u\end{cases}
$$

is continuous on $(0, T] \times \mathbb{R}$.

Remark 1. One can verify directly that $C(t, S)=e^{-r(T-t)} f_{u}\left(T-t, \log \left(S / S_{0}\right)\right)$ is just a different representation of (18). Recall that it gives the value of an up-and-out option with the payoff $H\left(S_{T}\right) 1_{T<\inf \left\{t \geq 0, S_{t} \geq U\right\}}$ at time $t$ when the stock price is $S$, if $S_{t}$ has not yet crossed the barrier (see (19)).

Proof. Since $H$ is Lipschitz, it is bounded on $(0, U)$. Let $M=\sup _{(0, U)} H(S)$.

We first show the continuity in $x$, for all $\tau>0$. If $x<u$ and $\varepsilon \in(0, u-x)$, we have

$$
\begin{aligned}
\left|f_{u}(\tau, x+\varepsilon)-f_{u}(\tau, x)\right| & =\left|\mathbb{E}\left[H\left(S_{0} e^{x+\varepsilon+Y_{\tau}}\right) 1_{\left\{\tau<R_{u-x-\varepsilon}\right\}}\right]-\mathbb{E}\left[H\left(S_{0} e^{x+Y_{\tau}}\right) 1_{\left\{\tau<R_{u-x}\right\}}\right]\right| \\
\leq \mid \mathbb{E}[( & \left.\left.H\left(S_{0} e^{x+\varepsilon+Y_{\tau}}\right)-H\left(S_{0} e^{x+Y_{\tau}}\right)\right) 1_{\left\{\tau<R_{u-x-\varepsilon}\right\}}\right] \mid+ \\
& +\left|\mathbb{E}\left[H\left(S_{0} e^{x+Y_{\tau}}\right) 1_{\left\{R_{u-x-\varepsilon} \leq \tau<R_{u-x}\right\}}\right]\right| \\
& \leq c S_{0} e^{x+r \tau}\left(e^{\varepsilon}-1\right)+M \mathbb{Q}\left[R_{u-x-\varepsilon} \leq \tau<R_{u-x}\right] \stackrel{\varepsilon \downarrow 0}{\longrightarrow} 0,
\end{aligned}
$$


by (31). We have used the martingale condition $\mathbb{E} e^{Y_{\tau}}=e^{r \tau}$ and the fact that $\tau<R_{u-x}$ implies $Y_{\tau} \leq u-x$, which is equivalent to $S_{0} e^{x+Y_{\tau}} \leq U$.

Similarly, for all $x<u$,

$$
\begin{aligned}
\left|f_{u}(\tau, x-\varepsilon)-f_{u}(\tau, x)\right| & =\left|\mathbb{E}\left[H\left(S_{0} e^{x-\varepsilon+Y_{\tau}}\right) 1_{\left\{\tau<R_{u-x+\varepsilon}\right\}}\right]-\mathbb{E}\left[H\left(S_{0} e^{x+Y_{\tau}}\right) 1_{\left\{\tau<R_{u-x}\right\}}\right]\right| \\
\leq \mid \mathbb{E}[( & \left.\left.H\left(S_{0} e^{x-\varepsilon+Y_{\tau}}\right)-H\left(S_{0} e^{x+Y_{\tau}}\right)\right) 1_{\left\{\tau<R_{u-x}\right\}}\right] \mid+ \\
& +\mathbb{E}\left[H\left(S_{0} e^{x-\varepsilon+Y_{\tau}}\right) 1_{\left\{R_{u-x} \leq \tau<R_{u-x+\varepsilon}\right\}}\right] \\
\leq & c S_{0} e^{x+r \tau}\left(1-e^{-\varepsilon}\right)+M \mathbb{Q}\left[R_{u-x} \leq \tau<R_{u-x+\varepsilon}\right] \stackrel{\varepsilon \downarrow 0}{\longrightarrow} 0,
\end{aligned}
$$

by (30). This proves the continuity of $f_{u}(\tau, \cdot)$ for all $x \neq u$.

The right continuity at $x=u$ is straightforward, since $f=0$ if $x \geq u$. It remains to verify the left continuity. For all $\tau>0$,

$$
\left|f_{u}(\tau, u-\varepsilon)-f_{u}(\tau, u)\right|=\left|\mathbb{E}\left[H\left(S_{0} e^{u-\varepsilon+Y_{\tau}}\right) 1_{\left\{\tau<R_{\varepsilon}\right\}}\right]\right| \leq M \mathbb{Q}\left[R_{\varepsilon}>\tau\right] \stackrel{\varepsilon \downarrow 0}{\longrightarrow} 0
$$

since $R_{0}=0$ almost surely. In consequence, $\forall \tau>0, f_{u}(\tau, \cdot)$ is continuous on $\mathbb{R}$. To show continuity in time, for $x<u$ and $t \geq s \geq 0$, we obtain:

$$
\begin{array}{r}
\left|f_{u}(t, x)-f_{u}(s, x)\right|=\left|\mathbb{E}\left[H\left(S_{0} e^{x+Y_{t}}\right) 1_{\left\{t<R_{u-x}\right\}}\right]-\mathbb{E}\left[H\left(S_{0} e^{x+Y_{s}}\right) 1_{\left\{s<R_{u-x}\right\}}\right]\right| \\
=\left|\mathbb{E}\left[\left(H\left(S_{0} e^{x+Y_{t}}\right)-H\left(S_{0} e^{x+Y_{s}}\right)\right) 1_{\left\{t<R_{u-x}\right\}}\right]-\mathbb{E}\left[H\left(S_{0} e^{x+Y_{s}}\right) 1_{\left\{s<R_{u-x} \leq t\right\}}\right]\right| \\
\leq c \mathbb{E}\left[S_{0} e^{x+Y_{s}}\left|e^{Y_{t-s}}-1\right| 1_{\left\{t<R_{u-x}\right\}}\right]+M \mathbb{Q}\left[s<R_{u-x} \leq t\right] \\
\leq c S_{0} e^{x+r s} \mathbb{E}\left|e^{Y_{t-s}}-1\right|+M \mathbb{Q}\left[s<R_{u-x} \leq t\right]
\end{array}
$$

The convergence of the first term to zero as $t \rightarrow s$ was already proven in Proposition 3. Let $t_{n} \downarrow s$ be an arbitrary sequence and denote $\Omega_{n}=\{\omega \in \Omega \mid s<$ $\left.R_{u-x}(\omega) \leq t_{n}\right\}$. Then, $\left\{\Omega_{n}\right\}$ is decreasing as $n \rightarrow \infty$ and

$\bigcap_{n>0} \Omega_{n}=\left\{\omega \in \Omega \mid \forall n, s<R_{u-x}(\omega) \leq t_{n}\right\} \subseteq\left\{\omega \in \Omega \mid s<R_{u-x}(\omega) \leq s\right\}=\emptyset$.

So, $\mathbb{Q}\left[s<R_{u-x} \leq t\right] \rightarrow 0$ as $t \rightarrow s$.

Remark 2. As the proof shows, if $\left\{Y_{t}\right\}$ is of type B or C, $f_{u}(\tau, x)$ is continuous on $(0, T] \times \mathbb{R} \backslash\{u\}$. If the condition $R_{0}=0$ a.s. is not satisfied, $f_{u}$ may be discontinuous at the barrier.

In order to study down-and-out options, let us define the process $\left\{R_{x}^{-}, x \geq 0\right\}$ of the first passage below a negative level:

$$
R_{x}^{-}=\inf \left\{s \geq 0 \mid Y_{s}<-x\right\}=\inf \left\{s \geq 0 \mid-Y_{s}>x\right\}
$$

It is clear that Lemmas $1-3$ apply to $R_{x}^{-}$provided $\left\{-Y_{t}\right\}$ satisfies the corresponding conditions. The generating triplet of $\left\{-Y_{t}\right\}$ being $(\sigma,-(r+\gamma), \nu(-d x))$, the dual process has the same type as $Y_{t}$ (in the sense of the Definition 1 ). However, note that $R_{0}=0$ a.s. does not imply $R_{0}^{-}=0$ a.s., as shows Table 1. 
Proposition 5. (Continuity of down-and-out options) Let $\left\{Y_{t}\right\}$ be of type $B$ or $C$ and $R_{0}^{-}=0$ a.s. Assume that $H:(L, \infty) \rightarrow[0, \infty)$ is Lipschitz:

$$
\forall S_{1}, S_{2} \in(L, \infty), \quad\left|H\left(S_{1}\right)-H\left(S_{2}\right)\right| \leq c\left|S_{1}-S_{2}\right|,
$$

with $L<S_{0}, c>0$ and denote $l=\log \left(L / S_{0}\right)$. Then, the function

$$
f_{l}(\tau, x)= \begin{cases}\mathbb{E}\left[H\left(S_{0} e^{x+Y_{\tau}}\right) 1_{\left\{\tau<R_{x-l}^{-}\right\}}\right], & x>l, \\ 0, & x \leq l,\end{cases}
$$

is continuous on $(0, T] \times \mathbb{R}$ ( $f_{l}$ represents the forward value of a down-and-out option with the payoff $\left.H\left(S_{T}\right) 1_{T<\inf \left\{t \geq 0, S_{t} \leq L\right\}}\right)$.

Proof. The proof is similar to the one of Proposition 4. The main difference is that $H$ may be unbounded, so we need to refine certain estimates.

To show the continuity of $f(\tau, \cdot)$ at $x>l$ (for a fixed $\tau>0$ ), we write:

$$
\begin{aligned}
&\left|f_{l}(\tau, x+\varepsilon)-f_{l}(\tau, x)\right|=\left|\mathbb{E}\left[H\left(S_{0} e^{x+\varepsilon+Y_{\tau}}\right) 1_{\left\{\tau<R_{x+\varepsilon-l}^{-}\right\}}\right]-\mathbb{E}\left[H\left(S_{0} e^{x+Y_{\tau}}\right) 1_{\left\{\tau<R_{x-l}^{-}\right\}}\right]\right| \\
& \leq \mid \mathbb{E}\left[\left(H\left(S_{0} e^{x+\varepsilon+Y_{\tau}}\right)-\right.\right.\left.\left.H\left(S_{0} e^{x+Y_{\tau}}\right)\right) 1_{\left\{\tau<R_{x-l}^{-}\right\}}\right] \mid+ \\
&+\mathbb{E}\left[H\left(S_{0} e^{x+\varepsilon+Y_{\tau}}\right) 1_{\left\{R_{x-l}^{-} \leq \tau<R_{x+\varepsilon-l}^{-}\right\}}\right] .
\end{aligned}
$$

The first term may be estimated as previously and goes to zero as $\varepsilon \downarrow 0$. For the second term we obtain:

$$
\begin{gathered}
\mathbb{E}\left[H\left(S_{0} e^{x+\varepsilon+Y_{\tau}}\right) 1_{\left\{R_{x-l}^{-} \leq \tau<R_{x+\varepsilon-l}^{-}\right\}}\right] \leq \mathbb{E}\left[C\left(1+S_{0} e^{x+\varepsilon+Y_{\tau}}\right) 1_{\left\{R_{x-l}^{-} \leq \tau<R_{x+\varepsilon-l}^{-}\right\}}\right] \\
\quad=C \mathbb{Q}\left[R_{x-l}^{-} \leq \tau<R_{x+\varepsilon-l}^{-}\right]+C S_{0} e^{x+\varepsilon+r \tau} \mathbb{E}\left[e^{Y_{\tau}} 1_{\left\{R_{x-l}^{-} \leq \tau<R_{x+\varepsilon-l}^{-}\right\}}\right] .
\end{gathered}
$$

The quantity $e^{Y_{\tau}} 1_{\left\{R_{x-l}^{-} \leq \tau<R_{x+\varepsilon-l}^{-}\right\}}$is bounded by an integrable variable $e^{Y_{\tau}}$ and converges to 0 in probability, since

$$
\forall \sigma>0, \quad \mathbb{Q}\left[e^{Y_{\tau}} 1_{\left\{R_{x-l}^{-} \leq \tau<R_{x+\varepsilon-l}^{-}\right\}}>\sigma\right] \leq \mathbb{Q}\left[R_{x-l}^{-} \leq \tau<R_{x+\varepsilon-l}^{-}\right] \stackrel{\varepsilon \downarrow 0}{\longrightarrow},
$$

by (30). Therefore, the dominated convergence theorem implies

$$
\mathbb{E}\left[e^{Y_{\tau}} 1_{\left\{R_{x-l}^{-} \leq \tau<R_{x+\varepsilon-l}^{-}\right\}}\right] \stackrel{\varepsilon \downarrow 0}{\longrightarrow} 0
$$

and the whole expression in (34) tends to zero as $\varepsilon \downarrow 0$. Using the same technique, one can show that $\left|f_{l}(\tau, x-\varepsilon)-f_{l}(\tau, x)\right| \rightarrow 0, \forall x>l$ and $\left|f_{l}(\tau, l-\varepsilon)\right| \rightarrow 0$, as $\varepsilon \downarrow 0$, which proves the continuity of $f_{l}$ in $x$. To show the continuity in time, we write for a fixed $x>l$ and $t \geq s \geq 0$ :

$$
\begin{aligned}
& \left|f_{l}(t, x)-f_{l}(s, x)\right|=\left|\mathbb{E}\left[H\left(S_{0} e^{x+Y_{t}}\right) 1_{\left\{t<R_{x-l}^{-}\right\}}\right]-\mathbb{E}\left[H\left(S_{0} e^{x+Y_{s}}\right) 1_{\left\{s<R_{x-l}^{-}\right\}}\right]\right| \\
& =\left|\mathbb{E}\left[\left(H\left(S_{0} e^{x+Y_{t}}\right)-H\left(S_{0} e^{x+Y_{s}}\right)\right) 1_{\left\{t<R_{x-l}^{-}\right\}}\right]-\mathbb{E}\left[H\left(S_{0} e^{x+Y_{s}}\right) 1_{\left\{s<R_{x-l}^{-} \leq t\right\}}\right]\right| \\
& \quad \leq c S_{0} e^{x+r s} \mathbb{E}\left|e^{Y_{t-s}}-1\right|+C \mathbb{Q}\left[s<R_{x-l}^{-} \leq t\right]+C S_{0} e^{x} \mathbb{E}\left[e^{Y_{s}} 1_{\left\{s<R_{x-l}^{-} \leq t\right\}}\right] .
\end{aligned}
$$

The convergence of the first two terms to zero, as $t \rightarrow s$, has already been proved and the last term can be treated in the same way as (35). 
Finally, we present a continuity result for double-barrier options. For $L<$ $S_{0}<U$, denote, as previously, $l=\log \left(L / S_{0}\right)$ and $u=\log \left(U / S_{0}\right)$.

Proposition 6. (Continuity of double-barrier options) Let $\left\{Y_{t}\right\}$ be of type $B$ or $C$, with $R_{0}=0$ and $R_{0}^{-}=0$ a.s. Assume that $H:(L, U) \rightarrow[0, \infty)$ is Lipschitz:

$$
\forall S_{1}, S_{2} \in(L, U), \quad\left|H\left(S_{1}\right)-H\left(S_{2}\right)\right| \leq c\left|S_{1}-S_{2}\right| .
$$

Then, the forward value of a double-barrier option with the payoff $H\left(S_{T}\right) 1_{T<\inf \left\{t \geq 0, S_{t} \notin(L, U)\right\}}$, defined by

$$
f_{d}(\tau, x)= \begin{cases}\mathbb{E}\left[H\left(S_{0} e^{x+Y_{\tau}}\right) 1_{\left\{\tau<R_{u-x} \wedge R_{x-l}^{-}\right\}}\right], & x \in(l, u), \\ 0, & x \notin(l, u),\end{cases}
$$

is continuous on $(0, T] \times \mathbb{R}$.

Proof. Let $M=\sup _{(L, U)} H(S)$. As in the two preceding propositions, we show the right and left continuity of $f_{d}$ at every point $x \in[l, u]$ using the Lemma 3. For example, $\forall \tau>0, \forall x \in(l, u)$,

$$
\begin{aligned}
& \left|f_{d}(\tau, x+\varepsilon)-f_{d}(\tau, x)\right|= \\
& =\left|\mathbb{E}\left[H\left(S_{0} e^{x+\varepsilon+Y_{\tau}}\right) 1_{\left\{\tau<R_{u-x-\varepsilon} \wedge R_{x+\varepsilon-l}^{-}\right\}}\right]-\mathbb{E}\left[H\left(S_{0} e^{x+Y_{\tau}}\right) 1_{\left\{\tau<R_{u-x} \wedge R_{x-l}^{-}\right\}}\right\}\right| \\
& \leq\left|\mathbb{E}\left[\left(H\left(S_{0} e^{x+\varepsilon+Y_{\tau}}\right)-H\left(S_{0} e^{x+Y_{\tau}}\right)\right) 1_{\left\{\tau<R_{u-x-\varepsilon} \wedge R_{x-l}^{-}\right\}}\right]\right|+ \\
& \quad+\mathbb{E}\left[H\left(S_{0} e^{x+\varepsilon+Y_{\tau}}\right) 1_{\left\{\tau<R_{u-x-\varepsilon}\right\}} 1_{\left\{R_{x-l}^{-} \leq \tau<R_{x+\varepsilon-l}^{-}\right\}}\right]+ \\
& \quad+\mathbb{E}\left[H\left(S_{0} e^{x+Y_{\tau}}\right) 1_{\left\{\tau<R_{x-l}^{-}\right\}} 1_{\left\{R_{u-x-\varepsilon} \leq \tau<R_{u-x}\right\}}\right] \\
& \leq c S_{0} e^{x+r \tau}\left(e^{\varepsilon}-1\right)+M \mathbb{Q}\left[R_{x-l}^{-} \leq \tau<R_{x+\varepsilon-l}^{-}\right]+M \mathbb{Q}\left[R_{u-x-\varepsilon} \leq \tau<R_{u-x}\right] \stackrel{\varepsilon \downarrow 0}{\longrightarrow} 0 .
\end{aligned}
$$

We do not give in detail the whole proof which follows the lines of Propositions 4 and 5 .

Interestingly, while investigating a different issue - the validity of smooth pasting conditions for American options in exp-Lévy models - Alili \& Kyprianou [1] arrive at conditions similar to the ones given in Propositions 4-5.

As shown by the examples above, in general one cannot hope for more than Lipschitz continuity with respect to the underlying. In particular, uniform bounds on derivatives, such as the ones required in [22], do not hold in cases of interest in finance - such as call or put options - where the payoff function $H$ is not smooth. In these cases, verification theorems such as Proposition 1 do not apply and the option pricing function should be seen as a viscosity solution of the PIDE (9).

\section{Option prices as viscosity solutions of PIDEs}

Existence and uniqueness of (classical) solutions for the PIDEs considered above in Sobolev / Hölder spaces have been studied in $[5,15]$ in the case where the 


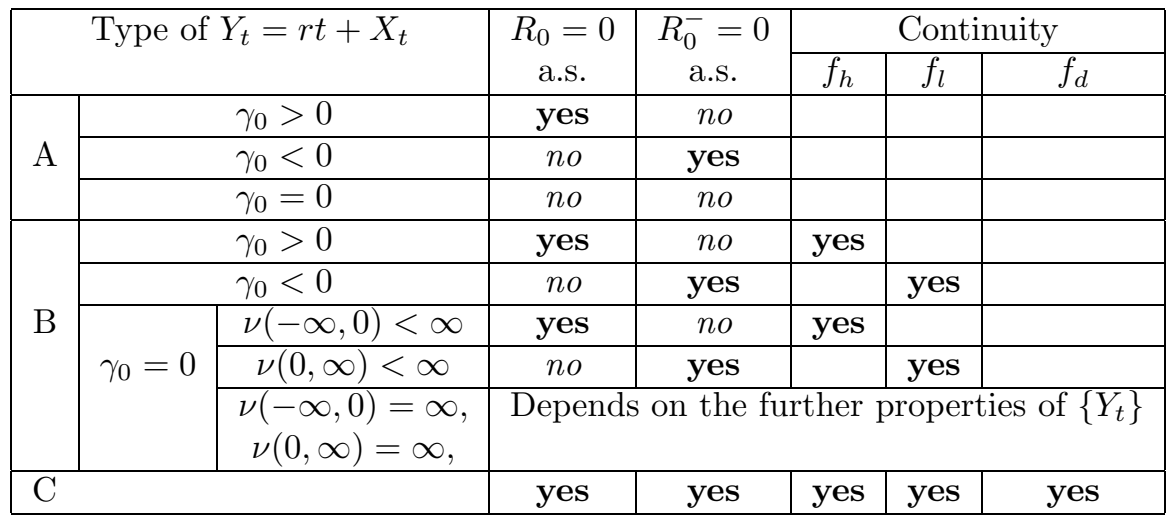

Table 1: This table shows the properties of $R_{0}$ and $R_{0}^{-}$for different types of Lévy processes and summarizes our results on the continuity of the barrier options. (An empty box does not mean the function is necessary discontinuous but there is no continuity result for this case). In the finite-variation case, $\gamma_{0}=r-\int\left(e^{y}-1\right) \nu(d y)$ is the drift.

diffusion component is non-degenerate: for a Lévy process this simply means $\sigma>0$ but more generally these results apply to jump diffusion where the diffusion coefficient is bounded away from zero. However many of the models in the financial modelling literature are pure jump models with $\sigma=0$, for which such results are not available. A notion of solution which yields both existence and uniqueness in this case is the notion of viscosity solution, introduced by Crandall \& Lions for PDEs (see e.g. [12] for a review) and extended to integro-differential equations of the type considered here in $[2,4,23,27,28] .^{3}$

\subsection{Viscosity solutions for PIDEs}

Denote by $U S C$ (respectively $L S C$ ) the class of upper semicontinuous (respectively lower semicontinuous) functions $v:(0, T] \times \mathbb{R} \rightarrow \mathbb{R}$ and by $C_{p}^{+}([0, T] \times \mathbb{R})$ the set of measurable functions on $[0, T] \times \mathbb{R}$ with polynomial growth of degree $p$ at plus infinity and bounded on $[0, T] \times \mathbb{R}^{-}$:

$$
\varphi \in C_{p}^{+}([0, T] \times \mathbb{R}) \Longleftrightarrow \exists C>0,|\varphi(t, x)| \leq C\left(1+|x|^{p} 1_{x>0}\right) .
$$

Under a polynomial decay condition on the right tail of the Lévy density, $L \varphi$ can be defined for $\varphi \in C^{2}([0, T] \times \mathbb{R}) \cap C_{p}^{+}([0, T] \times \mathbb{R})$ :

$$
\begin{aligned}
L \varphi(x) & =A \varphi(x)+\int_{|y| \leq 1} \nu(d y)\left[\varphi(x+y)-\varphi(x)-y \frac{\partial \varphi}{\partial x}(x)\right] \\
& +\int_{|y|>1} \nu(d y)[\varphi(x+y)-\varphi(x)],
\end{aligned}
$$

\footnotetext{
${ }^{3}$ Definitions of viscosity solutions in these papers vary in the choice of test functions; we present here a version which is suitable for option pricing applications.
} 
where $A$ is a differential operator. The terms in (38) are well defined for $\varphi \in$ $C^{2}([0, T] \times \mathbb{R})$ since

$$
\left|\varphi(\tau, x+y)-\varphi(\tau, x)-y \frac{\partial \varphi}{\partial x}(\tau, x)\right| \leq y^{2} \sup _{B(x, 1)}\left|\varphi^{\prime \prime}(\tau, \cdot)\right| \quad \text { for }|y| \leq 1,
$$

while the term in (39) is well defined for $\varphi \in C_{p}^{+}([0, T] \times \mathbb{R})$ since

$$
\int_{y>1} y^{p} \nu(d y)<+\infty
$$

due to the martingale condition (3).

Let $O=(l, u) \subseteq \mathbb{R}$ be an open interval, $\partial O=\{l, u\}$ its boundary, and $g \in C_{p}^{+}([0, T] \times \mathbb{R} \backslash O)$ a continuous function. Consider the following initialboundary value problem on $[0, T] \times \mathbb{R}$ :

$$
\begin{aligned}
\frac{\partial f}{\partial \tau}=L f+r \frac{\partial f}{\partial x}, & \text { on }(0, T] \times O, \\
f(0, x)=h(x), \quad x \in O ; & f(\tau, x)=g(\tau, x), \quad x \notin O .
\end{aligned}
$$

Definition 2 (Viscosity solution). A function $v \in U S C$ is a viscosity subsolution of (40)-(41) if for any test function $\varphi \in C^{2}([0, T] \times \mathbb{R}) \cap C_{p}^{+}([0, T] \times \mathbb{R})$ and any global maximum point $(\tau, x) \in[0, T] \times \mathbb{R}$ of $v-\varphi$, the following properties are verified:

$$
\begin{aligned}
\text { if }(\tau, x) \in(0, T] \times O, & \left(\frac{\partial \varphi}{\partial \tau}-L \varphi-r \frac{\partial \varphi}{\partial x}\right)(\tau, x) \leq 0, \\
\text { if } \tau=0, x \in \bar{O}, & \min \left\{\left(\frac{\partial \varphi}{\partial \tau}-L \varphi-r \frac{\partial \varphi}{\partial x}\right)(\tau, x), v(\tau, x)-h(x)\right\} \leq 0, \\
\text { if } \tau \in(0, T], x \in \partial O, & \min \left\{\left(\frac{\partial \varphi}{\partial \tau}-L \varphi-r \frac{\partial \varphi}{\partial x}\right)(\tau, x), v(\tau, x)-g(\tau, x)\right\} \leq 0, \\
\text { if } x \notin \bar{O}, & v(\tau, x) \leq g(\tau, x) .
\end{aligned}
$$

A function $v \in L S C$ is a viscosity supersolution of (40)-(41) if for any test function $\varphi \in C^{2}([0, T] \times \mathbb{R}) \cap C_{p}^{+}([0, T] \times \mathbb{R})$ and any global minimum point $(\tau, x) \in[0, T] \times \mathbb{R}$ of $v-\varphi$, we have:

$$
\begin{aligned}
\text { if }(\tau, x) \in(0, T] \times O, & \left(\frac{\partial \varphi}{\partial \tau}-L \varphi-r \frac{\partial \varphi}{\partial x}\right)(\tau, x) \geq 0, \\
\text { if } \tau=0, x \in \bar{O}, & \max \left\{\left(\frac{\partial \varphi}{\partial \tau}-L \varphi-r \frac{\partial \varphi}{\partial x}\right)(\tau, x), v(\tau, x)-h(x)\right\} \geq 0, \\
\text { if } \tau \in(0, T], x \in \partial O, & \max \left\{\left(\frac{\partial \varphi}{\partial \tau}-L \varphi-r \frac{\partial \varphi}{\partial x}\right)(\tau, x), v(\tau, x)-g(\tau, x)\right\} \geq 0, \\
\text { if } x \notin \bar{O}, & v(\tau, x) \geq g(\tau, x) .
\end{aligned}
$$

A function $v \in C_{p}^{+}([0, T] \times \mathbb{R})$ is called a viscosity solution of (40)-(41) if it is both a subsolution and a supersolution. $v$ is then continuous on $(0, T] \times \mathbb{R}$. 
A subsolution/supersolution need not be continuous and the initial and boundary conditions are verified in a generalized sense. The definition also includes the case of initial value problems: $O=\mathbb{R}$. Several variations on this definition can be found in the articles cited above. First, one can restrict the maximum/mimimum of $v-\varphi$ to be equal to zero:

Lemma 4. $v \in U S C$ is a viscosity subsolution of (40)-(41) if and only if for any $(\tau, x) \in[0, T] \times \mathbb{R}$ and any $\varphi \in C^{2}([0, T] \times \mathbb{R}) \cap C_{p}^{+}([0, T] \times \mathbb{R})$, properties

$$
v(\tau, x)=\varphi(\tau, x), \quad \text { and } \quad \forall(t, y) \in[0, T] \times \mathbb{R}, \quad v(t, y) \leq \varphi(t, y)
$$

imply (42)-(43).

Proof. Clearly, (44) means in particular that $(\tau, x)$ is a global maximum point of $v-\varphi$. Therefore, if $v$ is a subsolution then, by definition, (44) implies (42)-(43).

Conversely, if $v$ satisfies the condition of the lemma, we take a test function $\varphi$ and a global maximum point $(\tau, x)$ of $v-\varphi$, i.e.

$$
\forall(t, y) \in[0, T] \times \mathbb{R}, \quad v(t, y)-\varphi(t, y) \leq v(\tau, x)-\varphi(\tau, x),
$$

and need to show that $\varphi$ verifies (42)-(43). Define a new function $\psi$ by adding a constant to $\varphi$ :

$$
\psi(t, y)=\varphi(t, y)+[v(\tau, x)-\varphi(\tau, x)] .
$$

This function satisfies (44), so, by the condition, (42)-(43) are verified for $\psi$. But, $\forall(t, y) \in[0, T] \times \mathbb{R}$,

$$
\left(\frac{\partial \psi}{\partial \tau}-L \psi-r \frac{\partial \psi}{\partial x}\right)(t, y)=\left(\frac{\partial \varphi}{\partial \tau}-L \varphi-r \frac{\partial \varphi}{\partial x}\right)(t, y)
$$

which implies that the same properties are verified by $\varphi$, hence $v$ is a viscosity subsolution.

A similar result holds for supersolutions. Also, as shown in [4], one can replace "maximum" by "strict maximum". Finally, one can require the test functions to be $C^{1,2}$ or $C^{\infty}$ with bounded derivatives instead of $C^{2}$. The growth condition at infinity $\varphi \in C_{p}^{+}$on test functions is essential for $L \varphi$ to make sense. It may be replaced by other growth conditions under stronger hypotheses on the decay of the Lévy density.

Using the fact that $L$ verifies a maximum principle $[7,15]$, one can show that a classical solution $u \in C^{1,2}([0, T] \times \mathbb{R}) \cap C_{p}^{+}([0, T] \times \mathbb{R})$ is also a viscosity solution. However, since the definition above only involves applying derivatives to the test functions $\varphi$ and not to $u$, a viscosity solution need not be smooth: it is simply required to be continuous on $(0, T] \times \mathbb{R}$.

Unlike some other notions of generalized solution (such as weak solutions in Sobolev spaces), the pointwise value $u(t, x)$ is always defined for a viscosity solution: this is important since $u(t, x)$ corresponds to the option price. 
Remark 3 (Boundary conditions). We noted above that, for classical solutions, "boundary" conditions have to be imposed on $\mathbb{R} \backslash O$ and not only on the boundary $\partial O=\{l, u\}$. This seems not to be the case here since the non-local integral term only involves the test function and not the solution itself, so one can be led to think that conditions on the boundary are enough [23, Sec. 5.1.]. However note that the test functions have to verify $\varphi \geq v($ resp. $\varphi \leq v)$ on $[0, T] \times \mathbb{R}$ and not only on $[0, T] \times O$, which requires specifying $v$ outside $O$.

\subsection{Option prices as viscosity solutions of PIDE}

Existence and uniqueness of viscosity solutions for such parabolic integrodifferential equations are discussed in [2] in the case where $\nu$ is a finite measure and in $[4,23]$ and [18] for general Lévy measures. Growth conditions other than $u \in C_{p}^{+}$can be considered $[2,4]$ with additional conditions on the Lévy measure $\nu$. The main tool for showing uniqueness is the comparison principle: if $u$ are viscosity solutions and $u(0, x) \geq v(0, x)$ then $\forall \tau \in[0, T], u(\tau, x) \geq v(\tau, x)$. This property has been extended to subsolutions and supersolutions in [2] for the case where $\nu$ is a bounded measure; the case of a general Lévy measure has been recently treated in [18].

These results $[2,4,18]$ apply to viscosity solutions with polynomial growth at infinity. In the context of option prices, this restricts the choice of the payoff functions. We will give a sufficient condition on the payoff for the price to be in $C_{p}^{+}$(see (37) for the definition).

Lemma 5. For every $p \geq 0$ and $n \geq 1$, there exists $c>0$, such that

$$
\forall x_{1} \ldots x_{n} \geq 0, \quad\left(\sum_{i=1}^{n} x_{i}\right)^{p} \leq c \sum_{i=1}^{n} x_{i}^{p}
$$

Proof. If $p \geq 1$, it is Jensen's inequality with $g(x)=x^{p}$, and $c=n^{p-1}$. If $0 \leq p<1$, it is easily verified by induction with $c=1$.

Proposition 7. If $H:(0, \infty) \rightarrow[0, \infty)$ is Lipschitz: $\left|H\left(S_{1}\right)-H\left(S_{2}\right)\right| \leq C \mid S_{1}-$ $S_{2} \mid$, and there exists $p>0$, such that:

$$
H\left(S_{0} e^{x}\right) \leq C_{1}\left(1+|x|^{p}\right),
$$

then $f(\tau, x)=\mathbb{E}\left[H\left(S_{0} e^{x+r \tau+X_{\tau}}\right)\right]$ belongs to $C_{p}^{+}([0, T] \times \mathbb{R})$.

Proof. We first show that

$$
\mathbb{E}\left[\left(X_{\tau}\right)^{p} 1_{X_{\tau}>0}\right]<\infty .
$$

Theorem 25.3 of $[26]$ states that if $g: \mathbb{R} \rightarrow \mathbb{R}$ is a submultiplicative, locally bounded function, then $\mathbb{E} g\left(X_{\tau}\right)<\infty$ for all $\tau>0$ if and only if $\int_{|x|>1} g(x) \nu(d x)<\infty$.

A function $g(x) \geq 0$ is called submultiplicative if there exists $a>0$, such that

$$
\forall x, y \in \mathbb{R}, g(x+y) \leq a g(x) g(y) .
$$


A function is locally bounded if it is bounded on each compact.

For all $p>0$, the function $x^{p} \vee 1$ is submultiplicative [26, Prop. 25.4] and

$$
x^{p} \vee 1 \leq x^{p} 1_{x>0}+1 \leq 2\left(x^{p} \vee 1\right) .
$$

In consequence, for all $x, y \in \mathbb{R}$, we have

$$
\begin{aligned}
(x+y)^{p} 1_{x+y>0}+1 & \leq 2\left((x+y)^{p} \vee 1\right) \leq \\
& \leq 2 a\left(x^{p} \vee 1\right)\left(y^{p} \vee 1\right) \leq 2 a\left(x^{p} 1_{x>0}+1\right)\left(y^{p} 1_{y>0}+1\right),
\end{aligned}
$$

so, $g(x)=x^{p} 1_{x>0}+1$ is submultiplicative and locally bounded. By the theorem cited above, we obtain

$$
\mathbb{E}\left[\left(X_{\tau}\right)^{p} 1_{X_{\tau}>0}+1\right]<\infty \quad \Longleftrightarrow \int_{|x|>1}\left(x^{p} 1_{x>0}+1\right) \nu(d x)<\infty .
$$

Since $\nu$ is integrable on $|x|>1$, this clearly implies

$$
\mathbb{E}\left[\left(X_{\tau}\right)^{p} 1_{X_{\tau}>0}\right]<\infty \Longleftrightarrow \int_{x>1} x^{p} \nu(d x)<\infty .
$$

Thanks to the martingale condition, we have $\int_{x>1} e^{x} \nu(d x)<\infty$, and the condition on $\nu$ in (49) is satisfied all the more. So, (48) is also satisfied.

Since $H$ is Lipschitz, there exists $\tilde{c}>0$, such that $H(S) \leq \tilde{c}(1+S)$. Thus, for all $x \in \mathbb{R}$, we have

$$
f(\tau, x) \leq \tilde{c} \mathbb{E}\left[1+S_{0} e^{x+r \tau+X_{\tau}}\right]=\tilde{c}\left(1+S_{0} e^{x+r \tau}\right) .
$$

For the negative values of $x, f$ is bounded by the constant $\tilde{c}\left(1+S_{0} e^{r T}\right)$. Let $x>0$. We can estimate $f(\tau, x)$ in the following way:

$$
\begin{aligned}
& f(\tau, x)=\mathbb{E}\left[H\left(S_{0} e^{x+r \tau+X_{\tau}}\right) 1_{X_{\tau}<-x}\right]+\mathbb{E}\left[H\left(S_{0} e^{x+r \tau+X_{\tau}}\right) 1_{X_{\tau} \geq-x}\right] \\
& \quad \leq \tilde{c} \mathbb{E}\left[\left(1+S_{0} e^{x+r \tau+X_{\tau}}\right) 1_{X_{\tau}<-x}\right]+C_{1} \mathbb{E}\left[\left(1+\left(x+r \tau+X_{\tau}\right)^{p}\right) 1_{X_{\tau} \geq-x}\right] .
\end{aligned}
$$

The first term is bounded by $\tilde{c}\left(1+S_{0} e^{r T}\right)$, as previously. For the second, we obtain, using the Lemma 5:

$$
\begin{aligned}
& \mathbb{E}\left[\left(x+r \tau+X_{\tau}\right)^{p} 1_{X_{\tau} \geq-x}\right]= \\
& \quad=\mathbb{E}\left[\left(x+r \tau+X_{\tau}\right)^{p} 1_{\left|X_{\tau}\right| \leq x}\right]+\mathbb{E}\left[\left(x+r \tau+X_{\tau}\right)^{p} 1_{X_{\tau}>x}\right] \\
& \leq c\left(2 x^{p}+(r T)^{p}\right)+c\left(x^{p}+(r T)^{p}+\mathbb{E}\left[\left(X_{\tau}\right)^{p} 1_{X_{\tau} \geq 0}\right]\right) \leq C_{2}\left(1+x^{p}\right) .
\end{aligned}
$$

Putting this estimate into (51) gives

$$
f(\tau, x) \leq \tilde{c}\left(1+S_{0} e^{r T}\right)+C_{1}\left(1+C_{2}\left(1+x^{p}\right)\right) \leq C_{3}\left(1+x^{p}\right) .
$$

Corollary 1. If $H:(L, \infty) \rightarrow[0, \infty)$ is Lipschitz and satisfies the polynomial growth condition $(47)$, then $f_{l}(\tau, x)$ defined by $(33)$ is $C_{p}^{+}([0, T] \times \mathbb{R})$. 
Proof. Let us extend $H$ continuously on $(0, \infty)$ :

$$
\tilde{H}(S)= \begin{cases}H(S), & S>L \\ \lim _{S \rightarrow L} H(S), & S \leq L .\end{cases}
$$

Then, $\tilde{H}$ satisfies the conditions of Proposition 7, hence

$$
f_{l}(\tau, x) \leq \mathbb{E}\left[\tilde{H}\left(S_{0} e^{x+r \tau+X_{\tau}}\right)\right] \leq C\left(1+x^{p} 1_{x \geq 0}\right) .
$$

The following result shows that values of European and barrier options can be expressed as viscosity solutions of (40)-(41):

Proposition 8 (Option prices as viscosity solutions). Let the payoff function $H$ verify the Lipschitz condition (11) and let $h(x)=H\left(S_{0} e^{x}\right)$ have polynomial growth at infinity. Then:

- The forward value $f_{e}(\tau, x)$ of a European option defined by (8) is a viscosity solution of the Cauchy problem (9) (that is, (40)-(41) with $O=\mathbb{R}$ ).

- Let $f_{b}(\tau, x)$ be the forward value of a knockout (single or double) barrier option defined by (32), (33) or (36). If $f_{b}(\tau, x)$ is continuous on $(0, T] \times \mathbb{R}$ then it is a viscosity solution of (40)-(41) (with $g \equiv 0)$.

Proof. $f_{e}(\tau, x)$ is continuous by Proposition 3 and is $C_{p}^{+}([0, T] \times \mathbb{R})$ by Proposition 7. The functions $f_{u}$ and $f_{d}$ are bounded on $[0, T] \times \mathbb{R}$, and $f_{l}$ is $C_{p}^{+}([0, T] \times \mathbb{R})$ by the Corollary 1.

We will denote $f_{e}, f_{u}, f_{l}$, and $f_{d}$ by a generic name $f$, and $O$ will stand respectively for $\mathbb{R},(-\infty, u),(l, \infty)$ or $(l, u)$. So, $f$ is continuous and $C_{p}^{+}([0, T] \times$ $\mathbb{R}$ ), which is required in the definition of a viscosity solution. Let us now show that $f$ is a subsolution of (40)-(41). From the definition of $f$ it is easily seen that $f(0, x)=h(x)$ and $f(\tau, x)=0$ if $x \notin O$. Consider $\left(\tau_{0}, x_{0}\right) \in(0, T] \times O$ and a test function $\varphi \in C^{2}([0, T] \times \mathbb{R}) \cap C_{p}^{+}([0, T] \times \mathbb{R})$ such that

$$
\varphi\left(\tau_{0}, x_{0}\right)=f\left(\tau_{0}, x_{0}\right) \quad \varphi(\tau, x) \geq f(\tau, x) \quad \text { on }[0, T] \times \mathbb{R} .
$$

As noted in Section 4, we can suppose that $\left|\frac{\partial \varphi}{\partial \tau}\right|,\left|\frac{\partial \varphi}{\partial x}\right|$, and $\left|\frac{\partial^{2} \varphi}{\partial x^{2}}\right|$ are bounded by a constant $C$. Our goal is to show that $\varphi$ satisfies $(42)$ at $\left(\tau_{0}, x_{0}\right)$.

For $t \in\left[0, \tau_{0}\right]$, let $\theta_{t}=\inf \left\{s \geq t \mid x_{0}+Y_{s} \notin O\right\}$ where $Y_{s}=r s+X_{s}$. Define

$$
M_{t}=\mathbb{E}\left[H\left(S_{0} e^{x_{0}+Y_{\tau_{0}}}\right) 1_{\tau_{0} \leq \theta_{0}} \mid \mathcal{F}_{t}\right] .
$$

Note that $1_{\tau_{0} \leq \theta_{0}}=1_{\tau_{0} \leq \theta_{t}} 1_{t \leq \theta_{0}}$, and $1_{t \leq \theta_{0}} \in \mathcal{F}_{t}$. Since $Y_{s} \stackrel{d}{=} Y_{t}+Z_{s-t}, \forall s \geq t$, where $Z$ is a Lévy process independent of $Y$ and identically distributed, we can rewrite $M_{t}$ in the following way:

$$
\begin{aligned}
M_{t} & =1_{t \leq \theta_{0}} \mathbb{E}\left[H\left(S_{0} e^{\left(x_{0}+Y_{t}\right)+Z_{\tau_{0}-t}}\right) 1_{\tau_{0}-t \leq \inf \left\{s \geq 0,\left(x_{0}+Y_{t}\right)+Z_{s} \notin O\right\}} \mid \mathcal{F}_{t}\right] \\
& =1_{t \leq \theta_{0}} f\left(\tau_{0}-t, x_{0}+Y_{t}\right) \quad \text { a.s. }
\end{aligned}
$$


By construction, $M_{t}$ is a martingale. So, by the sampling theorem,

$$
f\left(\tau_{0}, x_{0}\right)=M_{0}=\mathbb{E}\left[M_{t \wedge \theta_{0}}\right]=\mathbb{E}\left[f\left(\tau_{0}-t \wedge \theta_{0}, x_{0}+Y_{t \wedge \theta_{0}}\right)\right],
$$

since $0 \leq \theta_{0}$ and $t \wedge \theta_{0} \leq \theta_{0}$. Then (52) implies, for all $t \in\left[0, \tau_{0}\right]$,

$$
f\left(\tau_{0}, x_{0}\right) \leq \mathbb{E}\left[\varphi\left(\tau_{0}-t \wedge \theta_{0}, x_{0}+Y_{t \wedge \theta_{0}}\right)\right] .
$$

Applying the Itô formula to the smooth function $\varphi\left(\tau_{0}-t, x_{0}+Y_{t}\right)$ between 0 and $t \wedge \theta_{0}$ gives:

$$
\begin{gathered}
f\left(\tau_{0}, x_{0}\right) \leq \varphi\left(\tau_{0}, x_{0}\right)+\mathbb{E}\left[\int_{0}^{t \wedge \theta_{0}}\left(-\frac{\partial \varphi}{\partial \tau}+L \varphi+r \frac{\partial \varphi}{\partial x}\right)\left(\tau_{0}-u, x_{0}+Y_{u_{-}}\right) d u\right] \\
+\mathbb{E}\left[\int_{0}^{t \wedge \theta_{0}} \frac{\partial \varphi}{\partial x}\left(\tau_{0}-u, x_{0}+Y_{u_{-}}\right) \sigma d W_{u}+\right. \\
\left.+\int_{0}^{t \wedge \theta_{0}} \int_{-\infty}^{\infty}\left(\varphi\left(\tau_{0}-u, x_{0}+Y_{u-}+y\right)-\varphi\left(\tau_{0}-u, x_{0}+Y_{u-}\right)\right) \tilde{J_{X}}(d u d y)\right],
\end{gathered}
$$

where $\tilde{J_{X}}$ is the compensated jump measure of $X$. The stochastic integral in (54) is a martingale (with zero expectation) if $\mathbb{E}\left[A_{t \wedge \theta_{0}}\right]<\infty$, where

$$
\begin{aligned}
A_{t}= & \int_{0}^{t}\left|\frac{\partial \varphi}{\partial x}\left(\tau_{0}-u, x_{0}+Y_{u_{-}}\right)\right|^{2} d u \\
& +\int_{0}^{t} d u \int_{-\infty}^{\infty} \nu(d y)\left|\varphi\left(\tau_{0}-u, x_{0}+Y_{u-}+y\right)-\varphi\left(\tau_{0}-u, x_{0}+Y_{u-}\right)\right|^{2} .
\end{aligned}
$$

Since $\varphi$ has bounded derivatives, $\mathbb{E}\left[A_{t \wedge \theta_{0}}\right]$ is bounded:

$$
\begin{aligned}
& \mathbb{E}\left[A_{t \wedge \theta_{0}}\right] \leq \mathbb{E}\left[\int_{0}^{t}\left|\frac{\partial \varphi}{\partial x}\left(\tau_{0}-u, x_{0}+Y_{u_{-}}\right)\right|^{2} d u+\right. \\
+ & \left.\int_{0}^{t} d u \int_{-\infty}^{\infty} \nu(d y) y^{2}\left|\frac{\partial \varphi}{\partial x}\left(\tau_{0}-u, x_{0}+Y_{u-}+\xi(y)\right)\right|^{2}\right] \leq C^{2} t\left(1+\int_{-\infty}^{\infty} y^{2} \nu(d y)\right) .
\end{aligned}
$$

Therefore, taking into account that $f\left(\tau_{0}, x_{0}\right)=\varphi\left(\tau_{0}, x_{0}\right)$, we derive from (54):

$$
\mathbb{E}\left[\int_{0}^{t} 1_{u \leq \theta_{0}}\left(\frac{\partial \varphi}{\partial \tau}-L \varphi-r \frac{\partial \varphi}{\partial x}\right)\left(\tau_{0}-u, x_{0}+Y_{u_{-}}\right) d u\right] \leq 0 .
$$

It is easily seen that the integrand is bounded, again by the boundedness of the derivatives of $\varphi$. Dividing (55) by $t$, taking the limit $t \rightarrow 0$, and applying the dominated convergence theorem, we finally obtain

$$
\left(\frac{\partial \varphi}{\partial \tau}-L \varphi-r \frac{\partial \varphi}{\partial x}\right)\left(\tau_{0}, x_{0}\right) \leq 0 .
$$


Hence, $f$ is a subsolution. Similarly, if $\varphi \in C^{2}([0, T] \times \mathbb{R}) \cap C_{p}^{+}([0, T] \times \mathbb{R})$ and $\left(\tau_{0}, x_{0}\right) \in(0, T] \times O$ are such that $\varphi\left(\tau_{0}, x_{0}\right)=f\left(\tau_{0}, x_{0}\right)$ and $\varphi \leq f$ on $[0, T] \times \mathbb{R}$, one can show that

$$
\left(\frac{\partial \varphi}{\partial \tau}-L \varphi-r \frac{\partial \varphi}{\partial x}\right)\left(\tau_{0}, x_{0}\right) \geq 0
$$

which implies that $f$ is a supersolution.

The hypotheses above on the payoff function apply to put options, singlebarrier knockout puts, double barrier knockout options and also to the logcontract. One can then retrieve call options by put-call parity.

\section{Discussion}

The characterization of option prices in terms of solutions of partial integrodifferential equations allows to use efficient numerical methods for pricing options on a single asset in presence of jumps. This relation has already been used by several authors to develop numerical methods for pricing options in models with jumps. In this paper we have shown that this characterization is less obvious in exponential Lévy model than in diffusion models, because of the possible lack of smoothness of option values with respect to the underlying in pure jump models. This lack of smoothness prevents the value function from being a classical solution of the pricing PIDE: we are led to use a notion of generalized solution. Using the notion of viscosity solution we have characterized in Proposition 8 the precise relation between PIDEs and prices of European or barrier options in exponential Lévy models. Such results are straightforward to extend to the case of time-dependent characteristics (additive processes) [9, Chapter 14]. ¿From the mathematical point of view one could also consider the case of state-dependent coefficients i.e. a general Markov process ("local volatility models with jumps") such as in [3]. However, as shown in [10], the addition of a local volatility component generates features which are redundant with the small jumps of the Lévy process and leads to an identification problem when calibrating the model to option prices. The gain from generality is therefore not clear and we have refrained from venturing in this direction.

The notion of viscosity solution turns out to be convenient for analyzing the convergence of finite difference schemes for PIDEs, without requiring smoothness with respect to the underlying. Such numerical methods are discussed in a companion paper [11]. The use of viscosity solutions allows to obtain pointwise convergence of option prices, which is more relevant for approximating option prices than $L^{2}$-type convergence obtained using the notion of weak solution in Sobolev spaces [21].

A key ingredient in the convergence is the comparison principle for semicontinuous solutions [2]. In principle, one can also define the notion of discontinuous viscosity (sub-/super-)solution for PIDEs, by replacing, in Definition $2, u$ by its (lower/upper) semicontinuous envelope. However, the comparison principle may fail to hold in this case and building convergent numerical schemes for computing such solutions may be a challenge. 


\section{References}

[1] L. Alili and A. Kyprianou, Some remarks on first passage of Lévy processes, the American put and pasting principles. 2004.

[2] O. Alvarez And A. Tourin, Viscosity solutions of non-linear integrodifferential equations, Annales de l'Institut Henri Poincaré, 13 (1996), pp. 293-317.

[3] L. Andersen And J. Andreasen, Jump-diffusion models: Volatility smile fitting and numerical methods for pricing, Rev. Derivatives Research, 4 (2000), pp. 231-262.

[4] G. Barles, R. Buckdahn, and E. Pardoux, Backward stochastic differential equations and integral-partial differential equations, Stochastics and Stochastic Reports, 60 (1997), pp. 57-83.

[5] A. Bensoussan and J.-L. Lions, Contrôle Impulsionnel et Inéquations Quasi-Variationnelles, Dunod, Paris, 1982.

[6] J. Bertoin, Lévy Processes, Cambridge University Press, Cambridge, 1996.

[7] J. Bony, Problème de Dirichlet et semi-groupe fortement fellerien associé à un opérateur integro-différentiel, Comptes Rendus de l'Académie des Sciences, 265 (1967), pp. 361-364.

[8] T. Chan, Pricing contingent claims on stocks driven by Lévy processes, Ann. Appl. Probab., 9 (1999), pp. 504-528.

[9] R. Cont and P. Tankov, Financial modelling with jump processes, Chapman \& Hall / CRC Press, 2003.

[10] R. Cont And P. Tankov, Nonparametric calibration of jump-diffusion option pricing models, Journal of Computational Finance, 7 (2004), pp. 149.

[11] R. Cont And E. Voltchkova, Finite difference methods for option pricing in jump-diffusion and exponential Lévy models, SIAM Journal on $\mathrm{Nu}-$ merical Analysis, (forthcoming).

[12] M. Crandall, H. IshiI, And P. Lions, Users guide to viscosity solutions of second order partial differential equations, Bulletin of the American Mathematical Society, 27 (1992), pp. 1-42.

[13] Y. D'Halluin, P. Forsyth, And G. Labahn, A penalty method for American options with jump-diffusion processes, working paper, University of Waterloo, March 2003.

[14] E. Eberlein, Applications of generalized hyperbolic Lévy motion to Finance, in Lévy Processes - Theory and Applications, O. Barndorff-Nielsen, T. Mikosch, and S. Resnick, eds., Birkhäuser, Boston, 2001, pp. 319-336. 
[15] M. Garroni and J. Menaldi, Second Order Elliptic Integro-Differential Problems, CRC Press, Boca Raton, FL, 2001.

[16] H. Geman, Pure jump Lévy processes for asset price modeling, Journal of Banking and Finance, 26 (2002), pp. 1297-1316.

[17] J. Jacod and A. N. Shiryaev, Limit Theorems for Stochastic Processes, Springer, Berlin, 2nd ed., 2002.

[18] E. Jakobsen and K. Karlsen, A maximum principle for semicontinuous functions applicable to integro-partial differential equations, working paper, Dept. of Mathematics, University of Oslo, 2003.

[19] D. Madan, Financial modeling with discontinuous price processes, in Lévy Processes-Theory and Applications, O. Barndorff-Nielsen, T. Mikosch, and S. Resnick, eds., Birkhäuser, Boston, 2001.

[20] D. Madan And F. Milne, Option pricing with variance gamma martingale components, Math. Finance, 1 (1991), pp. 39-55.

[21] A.-M. Matache, T. von Petersdorff, and C. Schwab, Fast deterministic pricing of options on Lévy driven assets, M2AN Math. Model. Numer. Anal., 38 (2004), pp. 37-71.

[22] D. Nualart And W. Schoutens, Backward stochastic differential equations and Feynman-Kac formula for Lévy processes, with applications in finance, Bernoulli, 7 (2001), pp. 761-776.

[23] H. Pham, Optimal stopping of controlled jump-diffusion processes: A viscosity solution approach, Journal of Mathematical Systems, 8 (1998), pp. 127 .

[24] P. Protter, Stochastic integration and differential equations, Springer, Berlin, 1990.

[25] S. RonG, On solutions of backward stochastic differential equations with jumps and applications, Stochastic Process. Appl., 66 (1997), pp. 209-236.

[26] K. Sato, Lévy Processes and Infinitely Divisible Distributions, Cambridge University Press, Cambridge, UK, 1999.

[27] A. SAYAh, Equations d'Hamilton Jacobi du premier ordre avec termes integro-differentiels, Comm. Partial Differential Equations, 16 (1991), pp. 1057-1093.

[28] H. Soner, Jump Markov Processes and Viscosity Solutions, vol. 10 of IMA Volumes in mathematics and applications, Springer Verlag, New York, 1986, pp. 501-511. 